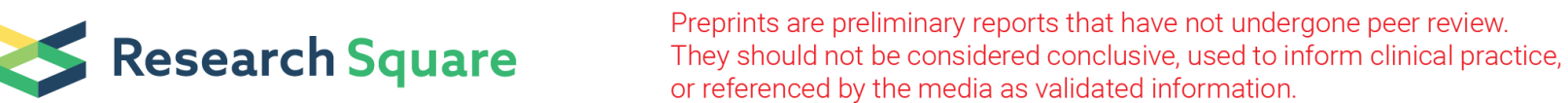

\section{PD-1 immune checkpoint blockade enhances FLOT chemotherapy toxicity in oesophageal adenocarcinoma cells via immune-independent mechanisms}

\section{maria davern}

Trinity St. James's Cancer Institute, Trinity College Dublin

\section{Rebecca M. O'Brien}

Trinity St. James's Cancer Institute, Trinity College Dublin Jason McGrath

Trinity St. James's Cancer Institute, Trinity College Dublin Noel E. Donlon

Trinity St. James's Cancer Institute, Trinity College Dublin Ashanty A. Melo

Trinity St. James's Cancer Institute, Trinity College Dublin Croí E. Buckley

Trinity St. James's Cancer Institute, Trinity College Dublin

Andrew D. Sheppard

Trinity St. James's Cancer Institute, Trinity College Dublin John V. Reynolds

Trinity St. James's Cancer Institute, Trinity College Dublin

Niamh Lynam-Lennon

Trinity St. James's Cancer Institute, Trinity College Dublin

\section{Stephen G. Maher}

Trinity St. James's Cancer Institute, Trinity College Dublin

Joanne Lysaght ( $\sim$ JLYSAGHT@tcd.ie )

Trinity St. James's Cancer Institute, Trinity College Dublin

\section{Research Article}

Keywords: PARP1, SMUG1, MMS19, nivolumab, atezolizumab, A2aR antagonism

Posted Date: October 25th, 2021

DOI: https://doi.org/10.21203/rs.3.rs-1000619/v1 
License: (c) (i) This work is licensed under a Creative Commons Attribution 4.0 International License. Read Full License 


\section{Abstract}

Chemotherapy upregulates immune checkpoint (IC) expression on the surface of tumour cells and ICintrinsic signalling confers a survival advantage against chemotherapy in several cancer-types including oesophageal adenocarcinoma (OAC). However, the signalling pathways mediating chemotherapyinduced IC upregulation and the mechanisms employed by ICs to protect OAC cells against chemotherapy remain unknown.

Longitudinal profiling revealed that FLOT-induced IC upregulation on OE33 OAC cells was sustained for up to 3 weeks post-treatment, returning to baseline upon complete tumour cell recovery. Pro-survival MEK signalling mediated FLOT-induced upregulation of PD-L1, TIM-3, LAG-3 and A2aR on OAC cells promoting a more immune-resistant phenotype. Single agent PD-1, PD-L1 and A2aR blockade decreased OAC cell viability, proliferation and mediated apoptosis. Mechanistic insights demonstrated that blockade of the PD-1 axis decreased stem-like marker ALDH and expression of DNA repair genes. Importantly, combining single agent PD-1, PD-L1 and A2aR blockade with FLOT enhanced cytotoxicity in OAC cells. These findings reveal novel mechanistic insights into the immune-independent functions of IC-intrinsic signalling in OAC cells with important clinical implications for boosting the efficacy of the first-line FLOT chemotherapy regimen in OAC in combination with ICB to not only boost anti-tumour immunity but also to suppress IC-mediated promotion of key hallmarks of cancer that drive tumour progression.

\section{Introduction}

Oesophageal adenocarcinoma (OAC) is the predominant subtype of oesophageal cancer in Western countries ${ }^{1}$. Moreover, OAC is an exemplar model of an obesity-driven cancer and as such its incidence is rapidly increasing in parallel with the rising level of obesity ${ }^{2}$. Response rates to the standard of care FLOT (5-fluorouracil (5-FU), oxaliplatin and docetaxel) chemotherapy regimen remain low with a complete pathologic response rate of $16.6 \%^{3}$ and subsequent clinical outcomes are dismal with a median overall survival rate of 50 months $^{3}$ and 5 -year overall survival rates as low as $15-40 \%$ depending on tumour stage at clinical presentation ${ }^{4}$. Improvements in the efficacy of first-line chemotherapy regimens were achieved through combining immune checkpoint blockade (ICB) with chemotherapy as depicted in the recent findings from the phase III Checkmate 649 trial, which demonstrated the synergy between nivolumab and first-line chemotherapy (FOLFOX and XELOX) in previously untreated OGJ patients $(n=1,581)$, in which a significant improvement in overall survival in patients with a PD-L1 combined positive score of 5 or greater was observed ( 14.4 months (nivolumab plus chemotherapy arm) vs. 11.1 months (chemotherapy arm) $)^{5}$. Furthermore, the nivolumab plus chemotherapy arm also reduced the risk of death by $29 \%(\mathrm{HR}, 0.71 ; 98.4 \% \mathrm{Cl}, 0.59-0.86 ; \mathrm{p}<0.0001)^{5}$. The findings from this trial highlight the potential synergy that can be exploited between chemotherapy and ICB in the first-line setting. Rational explanations for the improvement in overall survival are likely attributed to the ICB-mediated reinvigoration of anti-tumour immune responses. Moreover, mounting evidence in the literature suggest that the FOLFOX/XELOX chemotherapy regimens may possess immunostimulatory properties with the 
potential ability to convert a 'cold non-inflamed' into a 'hot inflamed' tumour microenvironment. 5fluorouracil and oxaliplatin chemotherapies which comprise the FLOT regimen, have been shown to induce immunogenic tumour cell death in several cancer types. Therefore, addition of ICB to the FLOT/XELOX regimen likely reinvigorated exhausted anti-tumour immunity and prevented exhaustion of chemotherapy-induced anti-tumour immune responses, producing synergistic amplification of antitumour immunity, which translated into durable clinical responses in gastroesophageal cancer patients. Oxaliplatin induced immunogenic cell death in colorectal cancer ${ }^{6}$ and lung carcinoma ${ }^{7,8}$, and 5-FU induced immunogenic cell death in colon carcinoma ${ }^{9}$ and gastric cancer ${ }^{10}$. Immunogenic cell death is a particular modality of cell death that can be triggered by selected anticancer chemotherapeutics ${ }^{11}$. Tumour cells undergoing immunogenic cell death are characterised by the release or exposure of damage-associated molecular patterns (DAMPs) that stimulate the attraction, activation and maturation of dendritic cells and eventually the antigen-specific priming of cytotoxic T lymphocytes ${ }^{12}$. This can induce an adaptive anticancer immune response that targets residual cancer cells with the same antigenic profile ${ }^{13}$.

Aside from ICB-mediated inhibition of immune evasion, another possible explanation of the beneficial outcomes with ICB may be attributed to their effect on immune-independent hallmarks of cancer. Abundant evidence in the literature has highlighted that use of ICB to inhibit PD-L $1^{14}, A 2 \mathrm{aR}^{15}$ or TIM-3 ${ }^{16}$ tumour cell-intrinsic signalling can suppress tumour cell invasion and migration and subsequent metastasis in a range of cancer models of gastric cancer, lung cancer and cervical cancer. Moreover, the PD-1 axis has been implicated in conferring chemo(radio)-resistance through promotion of stem-like characteristic in lung cancer ${ }^{17}$ and enhancement of radiation-induced DNA repair in osteosarcoma ${ }^{18}$. Additionally, PD-L1 signalling induced proliferation of tumour cells in a range of cancer types including hepatocellular carcinoma ${ }^{19}$, melanoma and ovarian cancer $^{20}$.

However, the mechanistic role of PD-1, PD-L1 and A2aR tumour cell-intrinsic signalling in the context of OAC remains to be investigated. Therefore, this study explores how the first-line FLOT chemotherapy regimen alters IC expression profiles of OAC cells longitudinally. The effect of single agent ICB on the viability of OAC cells is assessed including the ability of ICB to enhance FLOT chemotherapy cytotoxicity in OAC cells. Mechanistic insights are also provided surrounding the regulation of FLOT-induced upregulation of ICs on OAC cells and the effect of IC signalling on OAC cell proliferation, DNA repair and expression of cancer stem-like properties with and without FLOT treatment. Collectively, the findings of this study will offer important insights for the design of synergistic ICB-chemotherapy combinations for OAC patients.

\section{Methods}

Culture of cell lines

OE33 and SK-GT-4 oesophageal adenocarcinoma cells were purchased from European Collection of Cell Cultures. OE33 cells and SK-GT-4 cells were grown in RPMI 1640 medium with 2 mM L-glutamine 
(ThermoFisher Scientific, Ireland) and supplemented with 1\% (v/v) penicillin-streptomycin $(50 \mathrm{U} / \mathrm{ml}$ penicillin $100 \mu \mathrm{g} / \mathrm{ml}$ streptomycin (P/S)) and 10\% (v/v) foetal bovine serum (FBS) (ThermoFisher Scientific, Ireland) and detached using trypsin-EDTA solution (Gibco). All cell lines were maintained in a humidified chamber at $37^{\circ} \mathrm{C} 5 \% \mathrm{CO}_{2}$ and were tested regularly to ensure mycoplasma negativity. The OE33 cell line was established from a poorly differentiated stage IIA adenocarcinoma of the lower oesophagus (Barrett's metaplasia) of a 73-year old female patient. Whereas, the SK-GT-4 cell line originated from a well differentiated oesophageal adenocarcinoma arising in Barrett's epithelium from an 89-year-old Caucasian male.

Flow cytometry staining

OE33 cells and SK-GT-4 cells were treated with nivolumab $(10 \mu \mathrm{g} / \mathrm{ml}$, Opdivo), atezolizumab $(10 \mu \mathrm{g} / \mathrm{ml}$, Tecentriq), A2aR antagonist ( $3 \mu \mathrm{M}, \mathrm{ZM} 241385$ catalogue \# 1036/10 Bio-techne) or a MEK inhibitor $(0.001 \mu \mathrm{M}, \mathrm{U} 0126 \mathrm{Merck}, \mathrm{USA})$ in the absence or presence of FLOT chemotherapy regimen (5-FU, oxaliplatin and docetaxel). Doses of the FLOT chemotherapy regimen were pre-optimised to reduce the viability of cells by $50 \%$ ( $\mathrm{IC}_{50}$ dose) as previously described (OE33 cells: $0.8249 \mu \mathrm{M}$ 5-FU, $2 \mu \mathrm{M}$ oxaliplatin and $0.001 \mu \mathrm{M}$ of docetaxel and SK-GT-4 cells: $50 \mu \mathrm{M}$ 5-FU, $10 \mu \mathrm{M}$ oxaliplatin and $0.01 \mu \mathrm{M}$ docetaxel) or cells were treated with the vehicle control $\left(0.002 \% \text { DMSO, } 0.2 \% \mathrm{H}_{2} \mathrm{O}\right)^{21}$. Trypsinised OE33 cells or SK-GT-4 cells were stained with Zombie Aqua ${ }^{\mathrm{TM}}$, Violet ${ }^{\mathrm{TM}}$ or $\mathrm{NIR}^{\mathrm{TM}}$ viability dye (Biolegend, USA). Antibodies used for staining included LAG-3-FITC, CD160-PerCPCy5.5, PD-1-PE/Cy7, TIGIT-PE/Cy7 (Biolegend, USA), TIM-3AF647, PD-L1-FITC, PD-L2-PE (BD Bioscience, USA) and A2aR-PE (Bio-techne, USA). OAC cells were resuspended in FACs buffer and acquired using BD FACs CANTO II (BD Biosciences) using Diva software and analysed using FlowJo v10 software (TreeStar Inc.).

Detection of Ki67 staining by flow cytometry

Cells were collected by trypsinization, fixed in ice-cold 70\% ethanol (Merck, Darmstadt, Germany) and incubated at room temperature for 30 minutes. The fixative was decanted after centrifugation at 1,300 RPM for 3 mins and cells were resuspended in $100 \mu$ of FACs buffer and stained with $1 \mu$ of Ki67-AF647 (BioLegend). OAC cells were washed and resuspended in FACs buffer and acquired using BD FACs CANTO II (BD Biosciences) using Diva software and analysed using FlowJo v10 software (TreeStar Inc.).

Cell viability CCK-8 assay

A CCK-8 assay (Sigma, USA) was used to assess the effect of ICB with and without FLOT chemotherapy regimen on the proliferation rate of OE33 cells and SK-GT-4 cells. $5 \times 10^{3}$ OAC cells were adhered in a 96 well plate at $37^{\circ} \mathrm{C}, 5 \% \mathrm{CO} 2$ overnight. The media was removed, and cells were cultured for $48 \mathrm{~h}$ in complete RPMI in the absence or presence of nivolumab $(10 \mu \mathrm{g} / \mathrm{ml})$, atezolizumab $(10 \mu \mathrm{g} / \mathrm{ml})$, A2aR antagonist ( $3 \mu \mathrm{M}$, Bio-techne, USA) with and without the FLOT chemotherapy regimen $\left(\mathrm{IC}_{50}\right.$ dose). $5 \mu$ of CCK-8 solution was added to each well, followed by a $1.5 \mathrm{~h}$ incubation in the dark at $37^{\circ} \mathrm{C}, 5 \% \mathrm{CO} 2$. The optical density at $450 \mathrm{~nm}$ and $650 \mathrm{~nm}$ (reference wavelength) was measured using the Versa Max 
microplate reader (Molecular Devices, Sunnyvale, CA, USA) to determine a viable cell number. All of the data were analysed from three independent experiments.

Annexin $\mathrm{V}$ and propidium iodide assay

Apoptosis and necrosis was measured using annexin V (AV)-FITC and propidium iodide (PI) staining and was assessed by flow cytometry. OE33 cells and SK-GT-4 cells were cultured for $48 \mathrm{~h}$ in complete RPMI in the absence or presence of nivolumab $(10 \mu \mathrm{g} / \mathrm{ml})$, atezolizumab $(10 \mu \mathrm{g} / \mathrm{ml}), A 2 a R$ antagonist $(3 \mu \mathrm{M}$, Biotechne, USA) with and without the FLOT chemotherapy regimen ( $\left(\mathrm{C}_{50}\right.$ dose). Cells were stained with Annexin V-FITC (Biolegend, USA) and 1:4000 PI (Invitrogen, Carlsbad, CA, USA), and samples were acquired using BD FACs CANTO II (BD Biosciences) using Diva software and analysed using FlowJo v10 software (TreeStar, Inc., Ashland, Oregon).

Detection of $\mathrm{YH} 2 \mathrm{AX}$ by flow cytometry

After treatment, cells were collected by trypsinization, fixed in ice-cold 70\% ethanol (Merck, Darmstadt, Germany), and incubated at room temperature for 30 minutes. The fixative was decanted after centrifugation at 1,300 RPM for 3 mins with $2 \mathrm{ml}$ of PBS containing 2\% FBS. Cells were resuspended in $100 \mu \mathrm{l}$ H 2 AX staining (1:100 dilution of antibody) solution [Triton X100 (0.1\%), FBS (2\%)] for $2 \mathrm{~h}$ at room temperature. OAC cells were resuspended in FACs buffer and acquired using BD FACs CANTO II (BD Biosciences) using Diva software and analysed using FlowJo v10 software (TreeStar Inc.).

RNA isolation and quantification

Cells were seeded at a density of $3 \times 10^{6}$ cells in a T75 flask in $11 \mathrm{ml}$ of cRPMI and allowed to adhere overnight. Following drug treatment RNA was isolated from cell lines using the TRI Reagent ${ }^{\circledR}$ method. The RNA pellet was re-suspended in $30 \mu \mathrm{l}$ RNAase free molecular grade $\mathrm{H}_{2} \mathrm{O}$ and stored at $-80^{\circ} \mathrm{C}$. RNA quantification was determined spectrophotometrically, using a Nanodrop 1000 spectrophotometer (version 3.1.0, Nanodrop technologies, DE, USA).

cDNA synthesis

For cell line samples, total RNA ( $1 \mu \mathrm{g}$ total RNA) was reverse transcribed to cDNA using the manufacturer's instructions for the cDNA Reverse Transcription Kit from eBioscienes (4368814). In brief, to anneal the primers to the RNA, a master mix containing RNaseOUT 25X dNTP Mix $10 \mathrm{~mm},(10 \mathrm{mM}$, prepared as a 1:1:1:1 ratio of dATP, dGTP, dTTP and dCTP), 10x rt random primers, Bioscript reverse transcriptase (200units/ $\mu$ l) and 10X Bioscript Reaction Buffer in RNase-free water was added to each sample and the samples were incubated for 10 mins at $25^{\circ} \mathrm{C}$, for 120 mins at $37^{\circ} \mathrm{C}$ then for 5 mins at $85^{\circ} \mathrm{C}$ and held at $4^{\circ} \mathrm{C}$. The resulting cDNA was stored at $-20^{\circ} \mathrm{C}$.

Quantitative real time PCR and analysis 
qPCR was performed using TaqMan primer probes (MLH1, SMUG1, PARP1, MMS19 (Roche)) and a Quant Studio 5 real-time thermal cycler (Thermo Fisher Scientific). 18S (eBioscienes) was used as an endogenous control for data normalization. Data analysis was performed using Thermofisher Scientific Connect qPCR application software.

Aldehyde dehydrogenase (ALDH) assay

Aldehyde dehydrogenase (ALDH) enzyme activity was assessed using the Aldefluor ${ }^{\circledR}$ assay (Stem Cell Technologies), according to the manufacturer's instructions. Briefly, cells were trypsinised and resuspended at a density of $1 \times 10^{6}$ cells $/ \mathrm{mL}$ in Aldefluor ${ }^{\circledR}$ assay buffer containing ALDH substrate (bodipy-aminoacetaldehyde) $(5 \mu \mathrm{L} / \mathrm{mL})$. Immediately following this, half of the resuspended cells were added to a tube containing the ALDH inhibitor diethylaminobenzaldehyde (DEAB) to provide a negative control. Cells were acquired using BD FACs CANTO II (BD Biosciences) using Diva software and analysed using FlowJo v10 software (TreeStar Inc.).

\section{Statistical analysis}

Data were analysed using GraphPad Prism (GraphPad Prism, San Diego, CA, USA) and was expressed as mean \pm SEM. Statistical differences between two treatments in a particular cell line were analysed using a paired parametric Student's t-test. Statistical significance was determined as $p \leq 0.05$.

\section{Results}

Chemotherapy-induced upregulation of ICs on the surface of OAC cells is maintained up to 3 weeks posttreatment

We have previously demonstrated that first-line chemotherapy combinations FLOT and CROSS upregulated ICs on the surface of OAC cells following $48 \mathrm{~h}$ treatment in vitro ${ }^{21}$. Therefore, we sought to investigate how long this chemotherapy-induced upregulation of ICs on the surface of OAC cells is maintained by longitudinally profiling IC expression on the surface of OE33 cells following $48 \mathrm{~h}$ treatment with vehicle control or FLOT chemotherapy regimen (Figure 1A).

48h treatment with FLOT significantly increased PD-L1 expression on the surface of OE33 cells compared with the vehicle control at $48 \mathrm{~h}(50.85 \pm 1.2$ vs. $2.65 \pm 0.3 \%, p<0.0001$, Figure $1 \mathrm{~B})$. Interestingly, FLOTinduced PD-L1 upregulation on the surface of OE33 cells remained upregulated at 4 days ( $45.35 \pm 1.5$ vs. $1.63 \pm 0.5 \%, p<0.0001)$ and 21 days ( $8.88 \pm 0.3$ vs. $0.97 \pm 0.1 \%, p<0.0001$, Figure $1 B$ ) post-treatment compared with the vehicle control (Figure 1B). Following subculture of recovered FLOT-treated OE33 cells PD-L1 was significantly upregulated compared with the vehicle control $(3.15 \pm 0.2$ vs. $1.15 \pm 0.1, p=0.007$, Figure 1B).

Similarly, 48h treatment with FLOT significantly increased PD-L2 expression on the surface of OE33 cells compared with the vehicle control at $48 \mathrm{~h}$ (38.45 \pm 0.7 vs. $2.28 \pm 0.2 \%, p<0.0001$, Figure 1B). FLOT-induced 
PD-L2 upregulation on the surface of OE33 cells remained upregulated at 4 days ( $36.33 \pm 6.8$ vs. $2.5 \pm$ $0.9, p=0.01)$ and 21 days ( $3.7 \pm 0.1$ vs. $2.58 \pm 0.2, p=0.001$, Figure $1 B$ ) post-treatment compared with the vehicle control (Figure 1B). In contrast to PD-L1, following subculture of recovered (day 24) FLOT-treated OE33 cells PD-L2 expression had returned to baseline with no significant difference when compared with the vehicle control (Figure 1B).

Although 48h treatment with FLOT did not significantly alter CD160 expression on the surface of OE33 cells compared with the vehicle control at $48 \mathrm{~h}, \mathrm{CD} 160$ was significantly upregulated 4 days post-FLOT treatment compared with the vehicle control (73.53 \pm 5.6 vs. $3.75 \pm 0.7, p=0.0008$, Figure 1B). However, CD160 expression had returned to baseline 21 days post-FLOT treatment and following subculture of recovered FLOT-treated OE33 cells with no significant difference when compared with the vehicle control (Figure 1B).

Following 48h treatment with FLOT, PD-1 expression increased on the surface of OE33 cells on day 2 ( $46.18 \pm 4.3$ vs. $22.60 \pm 4.4, p=0.06)$ and on day 4 compared with the vehicle control $(28.95 \pm 7.5$ vs. $12.02 \pm 5.5 \%, p=0.06$, Figure $1 \mathrm{C}$ ). Furthermore, following subculture of recovered FLOT-treated OE33 cells there was a reduction in PD-1 expression compared with the vehicle control $(10.92 \pm 0.8$ vs. $17.63 \pm 0.8$, $\mathrm{p}=0.06$, Figure $1 \mathrm{C})$.

TIGIT expression increased on the surface of OE33 cells compared with the vehicle control at $48 \mathrm{~h}(68.58$ \pm 3.0 vs. $57.0 \pm 2.0 \%, p=0.06$, Figure $1 \mathrm{C})$ and remained upregulated at 4 days ( $67.28 \pm 6.8$ vs. $43.65 \pm 3.1$, $\mathrm{p}=0.01)$. However, 21 days post-FLOT treatment TIGIT expression returned to baseline and was comparable with the vehicle control (Figure 1C). Interestingly, following subculture of recovered FLOTtreated OE33 cells, TIGIT was significantly decreased compared with the vehicle control $(9.17 \pm 0.8 \mathrm{vs}$. $13.80 \pm 0.9 \%, p=0.004$, Figure $1 \mathrm{C})$.

Similar to TIGIT, following 48h treatment with FLOT, TIM-3 expression increased on the surface of OE33 cells compared with the vehicle control at $48 \mathrm{~h}(6.65 \pm 0.9$ vs. $1.8 \pm 0.1, \mathrm{p}=0.09$, Figure $1 \mathrm{C})$ and remained upregulated at 4 days (12.53 \pm 1.3 vs. $1.51 \pm 0.1 \%, p=0.003$ ) (Figure 1C). Similarly, to TIGIT, following subculture of recovered FLOT-treated OE33 cells was a significant reduction in TIM-3 expression compared with the vehicle control $(0.41 \pm 0.1$ vs. $1.95 \pm 0.4 \%, p=0.05$, Figure $1 \mathrm{C})$.

Following 48h treatment with FLOT, LAG-3 was significantly increased on the surface of OE33 cells compared with the vehicle control at $48 \mathrm{~h}(4.19 \pm 0.1$ vs. $1.90 \pm 0.4 \%, p=0.03$, Figure $1 \mathrm{C})$ and remained upregulated at 21 days ( $19.50 \pm 0.1$ vs. $4.43 \pm 0.3 \%, p=0.009$, Figure $1 \mathrm{C})$. Similar, to findings observed for TIGIT and TIM-3, following subculture of recovered FLOT-treated OE33 cells, LAG-3 expression decreased compared with the vehicle control ( $0.76 \pm 0.2$ vs. $1.71 \pm 0.4 \%, p=0.06$, Figure $1 \mathrm{C})$.

Although 48h treatment with FLOT did not significantly alter A2aR expression on the surface of OE33 cells compared with the vehicle control at $48 \mathrm{~h}, \mathrm{~A} 2 \mathrm{aR}$ was significantly upregulated 4 days post-FLOT treatment $(5.41 \pm 0.6$ vs. $1.41 \pm 0.2 \%, p=0.004)$ and 21 days $(1.78 \pm 0.1$ vs. $1.12 \pm 0.2 \%, p=0.02$, Figure 
1C). Similarly, following subculture of recovered FLOT-treated OE33 cells, A2aR expression was decreased compared with the vehicle control $(0.90 \pm 0.1$ vs. $2.16 \pm 0.4 \%, p=0.06$, Figure $1 \mathrm{C})$.

Overall, several ICs were significantly upregulated on the surface of OE33 cells longitudinally including PD-L1, PD-L2, CD160, TIGIT, TIM-3, LAG-3 and A2aR. Interestingly, PD-L1 remained increased compared with the vehicle control following subculture of FLOT-recovered OE33 cells at 24 days, whilst PD-L2 and CD160 returned to baseline expression levels. Interestingly, PD-1, TIGIT, TIM-3, LAG-3 and A2aR expression were decreased compared with the vehicle control following subculture of FLOT-recovered OE33 cells at 24 days.

\section{Pro-survival MEK signalling upregulates ICs on the surface of OAC cells following chemotherapy treatment}

Chemotherapy-induced upregulation of ICs on the surface of OAC cells suggests these tumour cells may be employing ICs as an adaptive survival mechanism to overcome genotoxic stress. However, the signalling pathways mediating FLOT-induced IC upregulation remain unknown. Therefore, we sought to investigate if the pro-survival signalling pathway MEK may be regulating the chemotherapy-induced upregulation of ICs.

Inhibition of MEK signalling significantly reduced the basal expression of PD-L1 on the surface of SK-GT4 cells compared with the vehicle control $(0.82 \pm 0.3$ vs. $2.13 \pm 0.1 \%, p=0.02)$ (Figure $2 A)$. Moreover, inhibition of MEK signalling significantly decreased FLOT-induced PD-L1 upregulation on the surface of OE33 cells ( $28.24 \pm 6.8$ vs. $31.63 \pm 7.1 \%, p=0.03)$ and SKGT-4 cells $(8.75 \pm 1.6$ vs. $31.38 \pm 6.5 \%, p=0.008)$ compared with FLOT treatment alone (Figure 2A).

Inhibition of MEK signalling significantly increased the expression of PD-L2 on the surface of OE33 cells ( $3.51 \pm 0.7$ vs. $1.72 \pm 0.1 \%, p=0.02)$ and SK-GT-4 cells $(5.79 \pm 0.9$ vs. $2.93 \pm 0.4 \%, p=0.001)$ compared with the vehicle control (Figure 2A). However, inhibition of MEK signalling did not alter the expression of FLOT-induced PD-L2 expression compared with FLOT treatment alone in either cell line.

Similarly, inhibition of MEK signalling significantly increased the expression of CD160 on the surface of OE33 cells ( $2.69 \pm 0.1$ vs. $1.4 \pm 0.1 \%, p=0.002)$ and SK-GT-4 cells $(1.32 \pm 0.1$ vs. $0.60 \pm 0.1 \%, p=0.04)$ compared with the vehicle control (Figure 2A). Moreover, inhibition of MEK signalling in combination with FLOT treatment significantly increased the expression of CD160 on the surface of OE33 cells compared with FLOT treatment alone (3.90 \pm 0.8 vs. $2.84 \pm 0.6 \%, p=0.03)$ compared with the vehicle control (Figure 2A).

Inhibition of MEK signalling significantly increased the expression of PD-1 on the surface of OE33 cells (11.15 \pm 2.5 vs. $6.97 \pm 1.3 \%, p=0.01)$ compared with the vehicle control (Figure 1B). In contrast, inhibition of MEK signalling significantly decreased the expression of PD-1 on the surface of SK-GT-4 cells (15.39 \pm 4.3 vs. $20.20 \pm 2.8 \%, p=0.001$ ) compared with the vehicle control (Figure $2 B$ ). Inhibition of MEK signalling 
did not significantly alter the expression of TIGIT on the surface of OE33 or SK-GT-4 cells basally or in combination with FLOT treatment (Figure 2B).

Inhibition of MEK signalling significantly reduced the expression of basal levels of TIM-3 on the surface of SK-GT-4 cells compared with the vehicle control ( $1.36 \pm 0.3$ vs. $2.26 \pm 0.2 \%, p=0.05)$ (Figure $2 A$ ). Inhibition of MEK signalling significantly decreased FLOT-induced TIM-3 upregulation on the surface of OE33 cells ( $3.92 \pm 0.2$ vs. $24.53 \pm 3.8 \%, p=0.03)$ and SKGT- 4 cells ( $3.35 \pm 0.3$ vs. $9.09 \pm 0.5 \%, p=0.001)$ compared with FLOT treatment alone (Figure $2 \mathrm{~A}$ ).

MEK inhibition significantly increased the expression of basal levels of LAG-3 on the surface of OE33 cells compared with the vehicle control ( $1.77 \pm 0.1$ vs. $0.96 \pm 0.1 \%, p=0.02)$ (Figure $2 A)$. In contrast, inhibition of MEK signalling significantly decreased FLOT-induced LAG-3 upregulation on the surface of SKGT-4 cells ( $5.80 \pm 0.8$ vs. $9.03 \pm 0.8 \%, p=0.002$ ) compared with FLOT treatment alone (Figure $2 A)$.

Additionally, inhibition of MEK signalling significantly decreased the expression of basal levels of A2aR on the surface of SK-GT-4 cells compared with the vehicle control (2.08 \pm 0.3 vs. $2.60 \pm 0.3, p=0.04)$ (Figure 2A). Similarly, inhibition of MEK signalling significantly decreased FLOT-induced A2aR upregulation on the surface of SKGT-4 cells $(4.47 \pm 0.9$ vs. $8.80 \pm 1.6, p=0.002)$ compared with FLOT treatment alone (Figure 2A). In addition, there were a reduction in FLOT-induced A2aR upregulation on the surface of OE33 cells compared with FLOT treatment alone ( $8.74 \pm 0.9$ vs. $16.83 \pm 2.7, p=0.06$, Figure $2 A)$.

Overall, MEK signalling regulated FLOT-induced upregulation of PD-L1, TIM-3, LAG-3 and A2aR on the surface of OAC cells. Of note, inhibition of the STAT3 signalling pathway did not significantly affect the expression of ICs on the surface of OAC cells (data not shown).

\section{Blockade of PD-L1, PD-1 and A2aR intrinsic signalling in OAC cells enhances the toxicity of the FLOT regimen}

Given the observation that the pro-survival MEK signalling pathway regulated FLOT-induced PD-L1 and A2aR upregulation on the surface of OAC cells, we investigated if blockade of PD-1 (nivolumab), PD-L1 (atezolizumab) or A2aR signalling axes in OAC cells might enhance the toxicity of FLOT chemotherapy regimen (Figure 3).

Single agent nivolumab and single agent A2aR antagonist significantly decreased the viability of OE33 cells compared with untreated cells $(84.12 \pm 5.2$ vs. $102.1 \pm 1.4 \%, p=0.02$ and $84.56 \pm 4.1$ vs. $102.4 \pm$ $1.8 \%, p=0.003$, respectively Figure $3 A$ ). Similar results were observed in the SK-GT-4 cell line; single agent nivolumab, single agent atezolizumab and single agent A2aR antagonist decreased the viability of SK-GT4 cells compared with untreated cells $(91.13 \pm 2.3$ vs. $100.0 \pm 0.9 \%, p=0.004,94.15 \pm 2.8$ vs. $100.0 \pm 0.9 \%$, $p=0.07$ and $91.70 \pm 2.7$ vs. $100.0 \pm 0.9 \%, p=0.008$, respectively Figure $3 A$ ).

Combining A2aR antagonist with FLOT significantly decreased the viability of OE33 cells compared with FLOT treatment alone $(71.69 \pm 3.1$ vs. $72.60 \pm 7.0 \%$, $p=0.03$, Figure $3 \mathrm{~A})$. Combining nivolumab with FLOT 
significantly decreased the viability of SK-GT-4 cells compared with FLOT treatment alone (36.01 \pm 4.4 vs. $38.72 \pm 2.7 \%, p=0.01$, Figure $3 \mathrm{~A}$ ).

Combining FLOT with nivolumab significantly decreased the viability of OE33 cells ( $51.57 \pm 14.50$ vs. $84.12 \pm 5.2 \%, p=0.02)$ and SK-GT-4 cells (36.01 \pm 4.4 vs. $91.13 \pm 2.3 \%, p<0.0001)$, compared with nivolumab treatment alone (Figure $3 \mathrm{~A}$ ). Combining FLOT with atezolizumab significantly decreased the viability of SK-GT-4 cells ( $37.61 \pm 3.8$ vs. $94.15 \pm 2.8 \%, p=0.0001)$, compared with atezolizumab treatment alone (Figure $3 \mathrm{~A}$ ). Combining FLOT with A2aR antagonism significantly decreased the viability of OE33 cells $(71.69 \pm 3.1$ vs. $84.56 \pm 4.1 \%, p=0.0005)$, and SK-GT-4 cells $(37.02 \pm 2.2$ vs. $91.70 \pm 2.7 \%, p<0.0001)$, compared with $\mathrm{A} 2 \mathrm{a} \mathrm{R}$ antagonism alone (Figure $3 \mathrm{~A}$ ).

Overall, single agent nivolumab and A2aR antagonism significantly decreased the viability of OAC cells alone. Interestingly, combining nivolumab or A2aR antagonist with the FLOT regimen significantly enhanced the reduction in viability of OAC cells compared with FLOT treatment alone. In addition, combining FLOT chemotherapy with single agent nivolumab, atezolizumab or A2aR antagonism significantly enhanced the reduction in viability of OAC cells compared with ICB treatment alone.

Given these findings we sought to investigate how blockade of the PD-1, PD-L1 or A2aR signalling axes alone and in combination with FLOT might affect the proliferation of OE33 cells longitudinally (Figure 3B). Single agent nivolumab significantly decreased Ki67 expression in OE33 cells at days 4 days (72.68 \pm 0.2 vs. $100.0 \pm 0.4 \%, p<0.0001)$ and 21 days $(71.07 \pm 0.2$ vs. $100.0 \pm 3.0 \%, p=0.01)$ compared with vehicle treated cells (Figure $3 \mathrm{C}$ ). Similarly, single agent atezolizumab significantly decreased Ki67 expression in OE33 cells 4 days ( $61.34 \pm 0.4$ vs. $100.0 \pm 3.0 \%, p<0.0001)$ and 21 days $(61.34 \pm 0.4$ vs. $100.0 \pm 3.0 \%, p=0.005$ ) compared with vehicle treated cells (Figure $3 C$ ). Single agent A2aR antagonist significantly increased Ki67 expression in OE33 cells at 4 days ( $139.9 \pm 0.3$ vs. $100.0 \pm 3.0 \%, p<0.0001)$ and decreased Ki67 expression at 21 days (63.68 \pm 0.2 vs. $100.0 \pm 3.0 \%, p=0.007)$ compared with vehicle treated cells (Figure 3C).

Interestingly, 48h FLOT treatment significantly increased Ki67 expression in OE33 cells compared with the vehicle control 2 days $(224.4 \pm 12.4$ vs. $100.0 \pm 4.0 \%, p=0.0008)$, 4 days ( $183.3 \pm 1.8$ vs. $100.0 \pm 0.4 \%$, $p<0.001)$ and 21 days $(145.95 \pm 5.2$ vs. $100.0 \pm 3.0 \%, p=0.002)$ post-treatment (Figure 3C). However, Ki67 expression was significantly decreased in the FLOT treated cells 21 days post-treatment compared with FLOT treated cells 4 days post-treatment ( $145.95 \pm 5.2$ vs. $183.3 \pm 1.8$ vs. $100.0 \pm 0.4 \%, p=0.01$ ) and compared with FLOT-treated cells 2 days post-treatment ( $145.95 \pm 5.2$ vs. $224.4 \pm 12.4 \%, p=0.03$ ) (Figure 3C).

Interestingly, single agent nivolumab in combination with FLOT treatment significantly decreased Ki67 expression in OE33 cells compared with FLOT treatment alone 2 days post-treatment (159.8 $\pm 6.1 \mathrm{vs}$. $224.4 \pm 12.36 \%, p=0.005)$ and 4 days post-treatment ( $170.3 \pm 0.4$ vs. $183.3 \pm 1.8 \%, p=0.003$ ) (Figure $3 \mathrm{C}$ ). Similarly, single agent atezolizumab in combination with FLOT treatment significantly decreased Ki67 expression in OE33 cells compared with FLOT treatment alone 2 days post-treatment (151.9 \pm 3.7 vs. $224.4 \pm 12.36 \%, p=0.02)$ and 21 days post-treatment (112.2 \pm 4.6 vs. $145.9 \pm 5.2 \%, p=0.02$ ) (Figure $3 \mathrm{C}$ ). 
Additionally, single agent A2aR antagonist in combination with FLOT treatment significantly decreased Ki67 expression in OE33 cells compared with FLOT treatment alone 2 days post-treatment $(155.8 \pm 5.8$ vs. $224.4 \pm 12.36 \%, p=0.02)$, 4 days post-treatment $(152.7 \pm 2.5$ vs. $183.3 \pm 1.8 \%, p=0.0001)$ and 21 days post-treatment $(80.49 \pm 4.6$ vs. $145.9 \pm 5.2 \%, p=0.0007)$ (Figure $3 C)$.

Overall, single agent nivolumab, atezolizumab and A2aR antagonism significantly decreased the proliferation of OAC cells alone. Interestingly, combining single agent nivolumab, atezolizumab and A2aR antagonism with the FLOT regimen significantly decreased the proliferation of OAC cells compared with FLOT treatment alone. Taken together these findings suggest that inhibition of the PD-1 axis or A2aR axis decreases the survival of OAC cells and when combined with the FLOT regimen synergistically enhance the toxicity of FLOT against OAC cells in vitro.

Given these findings we next aimed to investigate how blockade of PD-1, PD-L1 or A2aR signalling axes alone and in combination with FLOT might affect OAC cell apoptosis and cell death (Figure 4).

Single agent nivolumab significantly induced cell death in OE33 cells compared with the vehicle control (9.71 \pm 0.1 vs. $9.19 \pm 0.04 \%, p=0.02$ ), demonstrated by a significant increase in late stage apoptotic cells (Figure 4). Similarly, single agent A2aR antagonist significantly induced cell death in OE33 cells (19.25 \pm 0.2 vs. $9.19 \pm 0.04 \%, p<0.0001)$ and in SK-GT-4 cells $(6.88 \pm 0.1$ vs. $5.21 \pm 0.1 \%, p=0.001)$ compared with the vehicle control, demonstrated by a significant increase in late stage apoptotic cells (Figure 4). In addition, single agent nivolumab ( $9.13 \pm 0.3$ vs. $5.8 \pm 0.4 \%, p=0.005)$ and A2aR antagonist $(12.10 \pm 0.6$ vs. $5.8 \pm 0.4 \%, p=0.0004)$ significantly increased the percentage of early stage apoptotic SK-GT-4 cells compared with untreated cells (Figure 4).

Combining single agent nivolumab ( $23.78 \pm 2.4$ vs. $18.33 \pm 2.2 \%, p=0.04)$, atezolizumab $(27.23 \pm 4.1$ vs. $18.33 \pm 2.2 \%, p=0.04)$ or A2aR antagonist ( $28.45 \pm 0.8$ vs. $18.33 \pm 2.2 \%, p=0.04)$ with FLOT significantly induced cell death in OE33 cells compared with FLOT treated cells, demonstrated by a significant increase in late stage apoptotic cells (Figure 4). Similarly, combining single agent atezolizumab (10.28 \pm 0.4 vs. $8.44 \pm 0.3 \%, p=0.04)$ or A2aR antagonist ( $12.00 \pm 0.7$ vs. $8.44 \pm 0.3 \%, p=0.04)$ with FLOT significantly induced cell death in SK-GT-4 cells compared with FLOT treated cells, demonstrated by a significant increase in late stage apoptotic cells (Figure 4).

Although, combining single agent nivolumab with FLOT did not significantly enhance SK-GT-4 cell death, a significant increase in early stage apoptotic SK-GT-4 cells was observed using combination nivolumab with FLOT compared with FLOT alone ( $27.15 \pm 1.7$ vs. $13.73 \pm 1.6 \%, p=0.0002)$ (Figure 4). Similarly, combining single agent atezolizumab ( $27.15 \pm 1.7$ vs. $13.73 \pm 1.6 \%, p=0.001)$ or $A 2 a R$ antagonist $(24.90$ \pm 1.9 vs. $13.73 \pm 1.6 \%, p=0.0003$ ) with FLOT significantly induced increased the percentage of early stage apoptotic SK-GT-4 cells compared with FLOT treated cells (Figure 4).

Overall, these findings highlight that single agent PD-1, PD-L1 and A2aR IC blockade induced apoptosis and OAC cell death. Furthermore, combining ICB with the FLOT chemotherapy regimen synergistically enhanced induction of apoptosis in OAC cells and OAC cell death. 


\section{Blockade of IC signalling in OAC cells decreases the formation of $\mathrm{YH} 2 \mathrm{AX}$ and expression of DNA repair genes}

We have shown that PD-1, PD-L1 and A2aR signalling confers OAC cells with a survival advantage as their blockade alone reduces OAC cell viability and can enhance FLOT chemotherapy toxicity.

Interestingly, studies have implicated a role for PD-L1 intrinsic signalling in mediating DNA repair in colon cancer $^{22}$. Therefore, to achieve a greater understanding of the mechanisms of action behind enhanced FLOT cytotoxicity in combination with ICB we assessed if blockade of these IC pathways might alter the formation of $\mathrm{YH} 2 \mathrm{AX}$ alone and in combination with FLOT chemotherapy (Figure 5). Tumour cells rapidly proliferate and typically acquire DNA damage during replication generating genotoxic stress in the cells, which ultimately leads to tumour cell death if left unrepaired. Formation of $\mathrm{\gamma H} 2 \mathrm{AX}$ is an important step in the initiation of DNA repair.

Single agent nivolumab, atezolizumab and $\mathrm{A} 2 \mathrm{aR}$ antagonist significantly decreased the levels of $\mathrm{YH} 2 \mathrm{AX}$ expression in OE33 cells following $24 \mathrm{~h}$ treatment compared with the vehicle control (nivolumab: $1352 \pm$ 15.09 vs. $1507 \pm 8.51 \%, p=0.005$, atezolizumab: $1383 \pm 6.8$ vs. $1507 \pm 8.51 \%, p=0.002$ and $A 2 a R$ antagonist: $1416 \pm 13.6$ vs. $1507 \pm 8.51 \%, p=0.02$ ) (Figure $5 A$ ). Similar findings were observed in the SKGT-4 cell line where single agent nivolumab, atezolizumab and A2aR antagonist significantly decreased the levels of $\mathrm{YH} 2 \mathrm{AX}$ expression in OE33 cells following 24h compared with the vehicle control (nivolumab: $4167 \pm 50.85$ vs. $4491 \pm 32.9 \%, p=0.001$, atezolizumab: $4129 \pm 33.1$ vs. $4491 \pm 32.9 \%, p=0.002$ and $A 2 a R$ antagonist: $2791 \pm 38.6$ vs. $4491 \pm 32.9 \%, p<0.0001$ ) (Figure $5 \mathrm{~A}$ ).

Following 24h treatment with FLOT the levels of $\mathrm{YH} 2 \mathrm{AX}$ expression in OE33 cells was significantly increased compared with the vehicle control ( $2756 \pm 29.05$ vs. $1507 \pm 8.5 \%, p<0.0001)$. Interestingly, combining single agent nivolumab, atezolizumab and A2aR antagonist with the FLOT regimen significantly decreased the levels of $\mathrm{YH} 2 \mathrm{AX}$ expression in OE33 cells following 24h compared with FLOT treated cells (nivolumab: $1937 \pm 9.9$ vs. $2756 \pm 29.0 \%$, $p=0.0001$, atezolizumab: $1836 \pm 12.1$ vs. $2756 \pm$ $29.0 \%, p=0.0001$ and $A 2 a R$ antagonist: $2232 \pm 11.8$ vs. $2756 \pm 29.0 \%, p=0.0002$ ) (Figure $5 A$ ).

Similar findings were observed in the SK-GT-4 cell line (Figure 5A). Following 24h treatment with FLOT the levels of $\mathrm{YH} 2 \mathrm{AX}$ expression in SK-GT-4 cells was significantly increased compared with the vehicle control (5694 \pm 49.6 vs. $4491 \pm 32.9 \%, p=0.0001$ ). Similarly, combining single agent nivolumab with the FLOT regimen significantly decreased the levels of $\mathrm{YH} 2 \mathrm{AX}$ expression in SK-GT-4 cells following 24h compared with FLOT treated cells ( $4414 \pm 13.1$ vs. $5694 \pm 49.6 \%$, p<0.0001) (Figure $5 A)$. On the contrary, combining single agent nivolumab with the FLOT regimen significantly increased the levels of $\gamma \mathrm{H} 2 \mathrm{AX}$ expression in SK-GT-4 cells following 24h compared with FLOT treated cells (nivolumab: $4.44 \pm 4.1$ vs. $4.44 \pm 4.1 \%$, $\mathrm{p}<0.0001$ ) (Figure $5 \mathrm{~A}$ ). Overall, similar trends were also observed at $48 \mathrm{~h}$ and $\mathrm{ta} 72 \mathrm{~h}$ in which single agent nivolumab, atezolizumab and A2aR antagonism decreased $\mathrm{YH} 2 \mathrm{AX}$ expression in OE33 and SK-GT-4 cells compared with the vehicle control (Figure 5A). Similarly, at $48 \mathrm{~h}$ and $72 \mathrm{~h}$ timepoints, combining single agent nivolumab, atezolizumab and $A 2 a R$ antagonist with the FLOT regimen significantly decreased the levels of $\mathrm{YH} 2 \mathrm{AX}$ expression in OAC cells compared with FLOT treated cells (Figure 5A). 
Furthermore, Tu et al., ${ }^{23}$ demonstrated that intracellular PD-L1 acts as an RNA binding protein enhancing the mRNA stability of NBS1 and BRCA1, thus upregulating the expression of DNA repair proteins NBS1 and BRCA1. Therefore, we assessed if ICB might alter the expression of well described DNA repair genes PARP1, SMUG1, MLH1 and MMS19 alone and in combination with FLOT chemotherapy (Figure 5). Single agent atezolizumab significantly reduced the mRNA expression levels of PARP1 and SMUG1 compared with the vehicle control (PARP1: $0.41 \pm 0.1$ vs. $0.78 \pm 0.2 \%, \mathrm{p}=0.005$ and SMUG1: $0.45 \pm 0.1$ vs. $0.83 \pm$ $0.1 \%, p=0.008$ ) (Figure $5 B$ ). Interestingly, combining A2aR antagonist with the FLOT regimen significantly increased the mRNA expression levels of MMS19 compared with FLOT treated cells (1.12 \pm 0.1 vs. $0.13 \pm$ $0.06 \%, p=0.03$ ) (Figure 5B).

Overall, single agent nivolumab, atezolizumab and $\mathrm{A} 2 \mathrm{aR}$ antagonist decreased $\mathrm{\gamma H} 2 \mathrm{AX}$ expression in OAC cells and decreased the gene expression of DNA repair genes. Similarly, combining single agent nivolumab, atezolizumab with FLOT chemotherapy decreased $\mathrm{YH} 2 \mathrm{AX}$ expression in OAC cells and expression of DNA repair genes. Interestingly, although single agent A2aR antagonist decreased $\mathrm{yH} 2 \mathrm{AX}$ expression in OAC cells, an increase in expression of DNA repair genes was observed.

\section{Blockade of PD-1 axis signalling in OAC cells decreases ALDH stem-like marker}

Cancer stem-like cells exist as part of a subpopulation within tumours and are thought to be a major contributor to tumour recurrence. Moreover, our findings demonstrate that ICB decreases OAC cell proliferation and viability and induced OAC cell apoptosis and cell death in a subpopulation of OAC cells. Therefore, we aimed to investigate if ICB might be targeting the stem-like compartment within a population of OAC cells so we assessed the effect of ICB on the levels of ALDH stem-like marker in OAC cells alone and in combination with FLOT (Figure 6).

We found that single agent atezolizumab significantly decreased ALDH activity in OE33 cells compared with the vehicle control ( $28.44 \pm 2.5$ vs. $34.67 \pm 2.1 \%, p=0.004)$ (Figure 6). However, FLOT chemotherapy treatment significantly increased ALDH activity in OE33 cells compared with the vehicle control in OE33 cells (48.12 \pm 3.7 vs. $28.44 \pm 2.5 \%, p=0.01$ ) (Figure 6). Interestingly, nivolumab significantly attenuated the FLOT-induced increase in ALDH activity in OE33 cells compared with FLOT treated cells $(38.13 \pm 4.0$ vs. $48.12 \pm 3.7 \%, p=0.02$ ) (Figure 6).

Similarly, there were trends toward a significant decrease in ALDH activity following single agent atezolizumab treatment compared with the vehicle control in SK-GT-4 cells ( $3.39 \pm 1.3$ vs. $12.11 \pm 4.8 \%$, $\mathrm{p}=0.08$ ) (Figure 6). Overall, PD-L1 blockade decreased the expression of ALDH stem-like marker in OAC cells and PD-1 blockade attenuated the FLOT-induced increase in ALDH activity in OAC cells.

\section{Discussion}


We and others have previously shown that tumour cells express an array of both IC ligands and IC receptors ${ }^{21}$. Of particular clinical relevance we have previously shown that the FLOT chemotherapy regimen increases the expression of an array of ICs on the surface of OAC cells driving an immuneresistant phenotype within the tumour ${ }^{21}$. Furthermore, tumour cell-expressed ICs have also been implicated as a potential mechanism of chemo(radio)-resistance in a range of cancer types ${ }^{24-27}$. Firstly, by immune-dependent pathways via binding of ICs expressed on tumour cells with their cognate receptors or ligands on tumour-infiltrating immune cells negatively regulating anti-tumour function of these infiltrating immune cells ${ }^{28}$. Chemotherapy-induced PD-L1 upregulation on the surface of breast cancer cells results in immune evasion via ligation of PD-L1 to PD-1 on T cells, thereby inducing T cell apoptosis ${ }^{28}$. Alternatively, immune-independent mechanisms mediated by ICs have recently been recognised as potential mechanisms of resistance employed by tumour cells to attenuate the efficacy of chemo(radio)therapy efficacy. Such mechanisms include promotion of key hallmarks of cancer via ICintrinsic signalling in tumour cells including tumour cell metastasis ${ }^{15}$, enhanced DNA repair ${ }^{18}$, tumour cell proliferation ${ }^{20}$, cancer stem-like activity ${ }^{17}$ and glycolysis ${ }^{29}$ in tumour cells. Two cell lines were used in those study to help encapsulate the heterogeneity observed between tumours from different patients. The OE33 cell line was established from a poorly differentiated stage IIA adenocarcinoma which had progressed from Barrett's metaplasia in a 73-year old female patient. Whereas, the SK-GT-4 cell line was established from a well differentiated oesophageal adenocarcinoma which had also progressed from Barrett's metaplasia but from an 89-year-old male.

The findings from this study demonstrated that signalling through RAS/RAF/MEK/ERK (MAPK) signalling cascade upregulated several IC proteins including PD-L1, TIM-3, LAG-3 and A2aR on the surface of OAC cells following FLOT treatment. A complementary study demonstrated that MAPK signalling regulated epidermal growth factor- and interferon-gamma-induced PD-L1 expression in lung adenocarcinoma cells and that inhibition of MEK1/2 attenuated PD-L1 upregulation ${ }^{30}$. MEK signalling has pleiotropic effects in enhancing fundamental pro-tumourigenic processes, including tumour cell growth, survival and differentiation ${ }^{31}$. Collectively, these findings support a rationale for combination MAPK signalling and PD-L1 blockade to boost anti-tumour immunity as MAPK-induced upregulation of novel ICs such as TIM-3, LAG-3 and A2aR might represent mechanisms of immune escape or acquired resistance to PD-L1 ICB. A study in murine models further supports this rationale as dual MAPK inhibition in combination with anti-PD-L1 ICB resulted in synergistic and durable tumour regression even where either agent alone was only modestly effective ${ }^{32}$. The combination promoted $\mathrm{T}$ cell anti-tumour activity in combination with PD-L1 ICB ${ }^{32}$. Although MEK inhibition did profoundly block naive $\mathrm{CD} 8^{+} \mathrm{T}$ cell priming, an increased number of tumour-infiltrating, effector-phenotype, antigen-specific $\mathrm{CD} 8^{+} \mathrm{T}$ cells was observed $^{32}$. Furthermore, MEK inhibition protected tumour-infiltrating $\mathrm{CD} 8^{+} \mathrm{T}$ cells from chronic TCR stimulation-induced cell death while sparing cytotoxic activity ${ }^{32}$. MEK inhibition mediated downregulation of ICs on tumour cells may have also contributed to these synergistic effects. 
This study also profiled the dynamic alterations in the IC expression profiles of OAC cells post-FLOT treatment until complete recovery of OAC cells. Interestingly, IC expression was significantly upregulated in the immediate days post-FLOT treatment and was sustained longitudinally for up to 3 weeks on the surface of tumour cells. Upon complete recovery and subculture of tumour cells, we found that although PD-L1 expression had reduced substantially. expression remained significantly higher on the surface of post-FLOT tumour cells compared with untreated cells. Chemotherapy has been shown to select for a more treatment resistant tumour cell phenotype. Interestingly an isogenic model of cisplatin resistant lung cancer cells displayed significantly higher levels of PD-L1 expression compared with matched cisplatin sensitive cells ${ }^{33}$. Therefore, considering the findings of this study in context with the wider literature, the increased expression of PD-L1 on the surface of recovered OAC cells may suggest that PDL1 expression may identify but also confer a more treatment resistant phenotype.

PD-L1 upregulation is associated with activation of the DNA double-strand break repair pathway in patients with colon cancer ${ }^{22}$. Furthermore, several studies have shown that accumulation of damaged DNA and subsequent DNA damage signalling mediates upregulation of PD-L1 on the surface of tumour cells $^{34-36}$. In turn, additional studies have identified a role of PD-L1 tumour cell intrinsic signalling in enhancing the DNA damage response ${ }^{37}$. Tu et al., ${ }^{23}$ discovered that intracellular PD-L1 acts as an RNA binding protein that regulates the mRNA stability of NBS1 and BRCA1, thus enhancing repair of damaged DNA. In light of the mounting evidence that PD-L1 has a positive feedback regulatory role in the DNA damage response in cancer, our complementary findings further consolidate this novel immuneindependent role in OAC. We demonstrated that inhibition of the PD-1 signalling axis in OAC cells decreased expression of $\mathrm{YH} 2 \mathrm{AX}$, a surrogate marker of DNA damage which plays an important role in the DNA damage signalling response and subsequent repair of damaged $D N A^{38}$, and inhibited expression of DNA repair genes PARP1 and SMUG1 alone and in combination with FLOT.

Furthermore, elements of the PD-1 signalling axis namely the PD-1 receptor and its cognate ligand PD-L1 have been identified on the surface of stem-like tumour cells in melanoma ${ }^{39}$ and lung cancer ${ }^{17}$. We have previously shown that FLOT upregulates PD-L1 preferentially on the surface of stem-like OAC cells ${ }^{21}$. In addition, PD-1 and PD-L1 tumour cell intrinsic signalling has been shown to promote stem-like characteristics in both melanoma and lung cancer cells ${ }^{17,39}$. Similarly, the findings of this study demonstrated that blockade of the PD-1 signalling axis decreased ALDH stem-like marker in OAC cells. Collectively, these findings highlight an immune-independent role for the PD-1 axis signalling cascade in driving a treatment resistant phenotype, as cancer stem-like cells are thought to play a pivotal role in resistance to first-line chemotherapy regimens and subsequent tumour recurrence $e^{40,41}$.

FLOT comprises of a unique cocktail of chemotherapies including 5-FU (an anti-metabolite), oxaliplatin (DNA intercalator) and docetaxel (taxane) with specific mechanisms of action that target distinct phases of the cell cycle. 5-FU exerts its anticancer effects through inhibition of thymidylate synthase and incorporation of its metabolites into RNA and DNA resulting in damage, generating considerable amounts of cellular genotoxic stress and hindering the normal functioning and homeostasis of cellular processes 
that require these RNA and DNA ${ }^{42}$. Oxaliplatin, a platinum-based chemotherapy intercalates with cellular DNA forming platinum-DNA adducts, which induce DNA damage and block DNA replication ${ }^{43}$. Docetaxel inhibits microtubular depolymerization, and attenuation the effects of bcl-2 and bcl-xL gene expression which ultimately culminates in G2/M phase cell cycle arrest apoptotic cell death ${ }^{44}$. Although FLOT induced apoptosis within $48 \mathrm{~h}$ and decreased viability we also observed that FLOT increased the proliferation of OAC cells, however, the observed FLOT-induced proliferation of OAC cells was diminished longitudinally. Although this initial increase in proliferation was surprising the FLOT regimen may collectively hinder DNA damage signalling or repair and subsequent cell cycle arrest initially or may prevent delays through the cell cycle initially. This may subsequently cause the cells to undergo cell cycling at a faster rate increasing their proliferation initially, which then deceases longitudinally as the FLOT-treated cells die off in culture.

There are a number of studies in the literature highlighting that PD-1, PD-L1 and A2aR intrinsic signalling in tumour cells also promote tumour cell proliferation in a range of cancer types including hepatocellular carcinoma $^{19}$, lung, melanoma ${ }^{20}$, ovarian ${ }^{20}$, pancreatic ${ }^{45}$, gastric ${ }^{15,16,46}$ and cervical cancer ${ }^{16}$. The findings of this study further substantiate the immune-independent role of PD-1, PD-L1 and A2aR tumour cell-intrinsic signalling in promoting proliferation of tumour cells in the context of OAC.

In summary, the findings from this study highlight the novel immune-independent functions of IC tumour cell-intrinsic signalling in OAC cells promoting a range of hallmarks of cancer including promoting tumour cell growth and proliferation, enhancement of a cancer stem-like phenotype and enhancement of DNA repair. Importantly, blockade of the PD-1 signalling axis suppressed tumour cell growth, decreased cancer stem-like marker ALDH and expression of DNA repair genes alone and in combination with the FLOT chemotherapy regimen. Combining PD-L1, PD-1 or A2aR ICB with the FLOT regimen synergistically enhanced chemotherapy cytotoxicity in OAC cells. Overall, this highlights a strong clinical rational for combining ICB with the first-line chemotherapy regimens to not only reinvigorate anti-tumour immunity and prevent immune exhaustion but to directly enhance the cytotoxicity of FLOT via inhibition of immune-independent hallmarks of cancer mediated by IC-intrinsic signalling in OAC cells.

\section{Declarations}

Specific author contributions

All authors have contributed significantly to this manuscript. Maria Davern carried out the investigative techniques, performed data analysis, interpreted the results and contributed to conception and design of the study. Jason McGrath, Noel E. Donlon and Andrew D. Sheppard carried out investigative techniques. Rebecca M. O' Brien and Croí E. Buckley assisted with experiments. Ashanty A. Melo, Stephen G. Maher, Niamh Lynam-Lennon and John V. Reynolds contributed to design of the project. Joanne Lysaght made substantial contribution to the conception and design of the project, and interpretation of the data and overall supervision of the project. All authors were involved in the drafting and critical appraisal of the manuscript and have given approval of the final version for publication. 
Financial disclosure

This work was funded by an Irish Research Council scholarship (GOIPG/2017/1659) and the CROSS oesophageal cancer research charity.

Conflicts of Interest

The authors declare that there is no conflict of interest that could be perceived as prejudicing the impartiality of the research reported.

\section{Acknowledgements}

Thank you to the Beacon Hospital for gifting us with nivolumab and atezolizumab.

\section{References}

1. Pera, M., Manterola, C., Vidal, O. \& Grande, L. Epidemiology of esophageal adenocarcinoma. J. Surg. Oncol, 92, 151-159 (2005).

2. Kubo, A., Corley, D. A., Jensen, C. D. \& Kaur, R. Dietary factors and the risks of oesophageal adenocarcinoma and Barrett's oesophagus. Nutr. Res. Rev, 23, 230-246 (2010).

3. Villanueva, L. et al. Total neoadjuvant chemotherapy with FLOT scheme in resectable adenocarcinoma of the gastro-oesophageal junction or gastric adenocarcinoma: Impact on pathological complete response and safety. Ecancermedicalscience, 15, 1-10 (2020).

4. Huang, F. L. \& Yu, S. J. Esophageal cancer: Risk factors, genetic association, and treatment. Asian J. Surg, 41, 210-215 (2018).

5. Moehler, M. H. et al. CheckMate 649: A randomized, multicenter, open-label, phase III study of nivolumab (NIVO) + ipilimumab (IPI) or nivo + chemotherapy (CTX) versus CTX alone in patients with previously untreated advanced (Adv) gastric (G) or gastroesophageal junction (GEJ). J. Clin. Oncol, 36, TPS192-TPS192 (2018).

6. Wang, R., Yu, J. \& Zhang, L. Y. J.. F. Immunogenic effects of chemotherapy-induced tumor cell death. Genes Dis, 5, 194-203 (2018).

7. Sun, L., Li, T., Chen, S., Song, J. \& Li, D. F.. C. Oxaliplatin induces immunogenic cells death and enhances therapeutic efficacy of checkpoint inhibitor in a model of murine lung carcinoma. J Recept Signal Transduct Res, 39, 208-214 (2019).

8. Haruna, M. et al. M.. H. Docetaxel Upregulates HMGB1 Levels in Non-small Cell Lung Cancer. Biol Pharm Bull, 43, 399-403 (2020).

9. Cottone, A. et al. L.. C. 5-Fluorouracil causes leukocytes attraction in the peritoneal cavity by activating autophagy and HMGB1 release in colon carcinoma cells. Int J Cancer, 136, 1381-1389 (2015). 
10. Galetto, S., Forno, S., Moro, F., Mussa, A. \& Matera, L. A.. B. Drug- and cell-mediated antitumor cytotoxicities modulate cross-presentation of tumor antigens by myeloid dendritic cells. Anticancer Drugs, 14, 833-843 (2003).

11. Galluzzi, L., Buqué, A., Kepp, O., Zitvogel, L. \& Kroemer, G. Immunogenic cell death in cancer and infectious disease. Nat. Rev. Immunol, 17, 97-111 (2017).

12. Serrano-del Valle, A., Anel, A., Naval, J. \& Marzo, I. Immunogenic Cell Death and Immunotherapy of Multiple Myeloma. Frontiers in Cell and Developmental Biology, 7, 50 (2019).

13. Kroemer, G., Galluzzi, L., Kepp, O. \& Zitvogel, L. Immunogenic Cell Death in Cancer Therapy. Annu. Rev. Immunol, 31, 51-72 (2013).

14. Hsu, W. et al. J. M.. X. STT3-dependent PD-L1 accumulation on cancer stem cells promotes immune evasion. Nat Commun, 9, 1908 (2018).

15. Shi, Z. et al. L.. W. Adenosine interaction with adenosine receptor A2a promotes gastric cancer metastasis by enhancing PI3K-AKT-mTOR signaling. Mol Biol Cell, 30, 2527-2534 (2019).

16. Cao, Y. et al. Tim-3 expression in cervical cancer promotes tumor metastasis. PLoS One, 8, e53834e53834 (2013).

17. Raniszewska, A., Polubiec-Kownacka, M., Rutkowska, E. \& Domagala-Kulawik, J. PD-L1 Expression on Lung Cancer Stem Cells in Metastatic Lymph Nodes Aspirates. Stem Cell Rev. Reports, 15, 324330 (2019).

18. Sato, H. et al. DNA double-strand break repair pathway regulates PD-L1 expression in cancer cells. Nat. Commun, 8, 1751 (2017).

19. Zhong, F., Cheng, X., Sun, S. \& Zhou, J. Transcriptional activation of PD-L1 by Sox2 contributes to the proliferation of hepatocellular carcinoma cells. Oncol. Rep, 37, 3061-3067 (2017).

20. Gupta, H. B. et al. Tumor cell-intrinsic PD-L1 promotes tumor-initiating cell generation and functions in melanoma and ovarian cancer. Signal Transduct. Target. Ther, 1, 16030 (2016).

21. Davern, M. et al. Chemotherapy regimens induce inhibitory immune checkpoint protein expression on stem-like and senescent-like oesophageal adenocarcinoma cells. Transl. Oncol, 14, 101062 (2021).

22. Ozawa, N. et al. PD-L1 upregulation is associated with activation of the DNA double-strand break repair pathway in patients with colitic cancer. Sci. Rep, 11, 13077 (2021).

23. Tu, X. et al. PD-L1 (B7-H1) Competes with the RNA Exosome to Regulate the DNA Damage Response and Can Be Targeted to Sensitize to Radiation or Chemotherapy. Mol. Cell, 74, 1215-12264 (2019).

24. Liu, N. et al. Programmed death 1 induces cell chemoresistance to 5 -fluorouracil in gastric cancer cell lines. Transl. Cancer Res, 5, 781-788 (2016).

25. Sato, A. et al. H.. N. DNA double-strand break repair pathway regulates PD-L1 expression in cancer cells. Nat Commun, 8, 1751 (2017).

26. Permata, Y. et al. T. B. M.. H. Base excision repair regulates PD-L1 expression in cancer cells., 38, 4452-4466 (2019). 
27. Tu, B. et al. X.. Q. PD-L1 (B7-H1) Competes with the RNA Exosome to Regulate the DNA Damage Response and Can Be Targeted to Sensitize to Radiation or Chemotherapy. Mol Cell 74, 12151226.e4(2019).

28. Zhang, P., Su, D. M., Liang, M. \& Fu, J. Chemopreventive agents induce programmed death-1-ligand 1 (PD-L1) surface expression in breast cancer cells and promote PD-L1-mediated T cell apoptosis. Mol. Immunol, 45, 1470-1476 (2008).

29. Kim, S. et al. Programmed cell death ligand-1-mediated enhancement of hexokinase 2 expression is inversely related to T-cell effector gene expression in non-small-cell lung cancer. J. Exp. Clin. Cancer Res, 38, 462 (2019).

30. Stutvoet, T. S. et al. MAPK pathway activity plays a key role in PD-L1 expression of lung adenocarcinoma cells. J. Pathol, 249, 52-64 (2019).

31. Degirmenci, U., Wang, M., Hu, J. \& Targeting Aberrant RAS/RAF/MEK/ERK Signaling for Cancer Therapy.Cells9, (2020).

32. Ebert, P. J. R. et al. MAP Kinase Inhibition Promotes T Cell and Anti-tumor Activity in Combination with PD-L1 Checkpoint Blockade., 44, 609-621 (2016).

33. Yan, F. et al. Elevated Cellular PD1/PD-L1 Expression Confers Acquired Resistance to Cisplatin in Small Cell Lung Cancer Cells. PLoS One, 11, e0162925 (2016).

34. Sato, H. et al. DNA double-strand break repair pathway regulates PD-L1 expression in cancer cells. Nat. Commun, 8, 1751 (2017).

35. Permata, T. B. M. et al. Base excision repair regulates PD-L1 expression in cancer cells., 38, 44524466 (2019).

36. Sato, H., Jeggo, P. A. \& Shibata, A. Regulation of programmed death-ligand 1 expression in response to DNA damage in cancer cells: Implications for precision medicine. Cancer Sci, 110, 3415-3423 (2019).

37. Meng, X., Yang, S. \& Camp, V. J. A. The Interplay Between the DNA Damage Response, RNA Processing and Extracellular Vesicles. Frontiers in Oncology, 9, 1538 (2020).

38. Mah, L. J., El-Osta, A. \& Karagiannis, T. C. yH2AX: a sensitive molecular marker of DNA damage and repair., 24, 679-686 (2010).

39. Kleffel, S. et al. Melanoma Cell-Intrinsic PD-1 Receptor Functions Promote Tumor Growth., 162, 1242-1256 (2015).

40. Wang, Q. E. DNA damage responses in cancer stem cells: Implications for cancer therapeutic strategies. World J. Biol. Chem, 6, 57-64 (2015).

41. Reya, T., Morrison, S. J., Clarke, M. F. \& Weissman, I. L. Stem cells, cancer and cancer stem cells. Nature, 414, 105-111 (2001).

42. Longley, D. B., Harkin, D. P. \& Johnston, P. G. 5-Fluorouracil: mechanisms of action and clinical strategies. Nat. Rev. Cancer, 3, 330-338 (2003). 
43. Martinez-Balibrea, E. et al. Tumor-Related Molecular Mechanisms of Oxaliplatin Resistance. Mol. Cancer Ther. 14, 1767 LP - 1776(2015).

44. Pienta, K. J. Preclinical mechanisms of action of docetaxel and docetaxel combinations in prostate cancer. Semin. Oncol, 28, 3-7 (2001).

45. Gao, M. et al. Direct therapeutic targeting of immune checkpoint PD-1 in pancreatic cancer. Br. J. Cancer, 120, 88-96 (2019).

46. Jiang, J. et al. Decreased galectin-9 and increased Tim-3 expression are related to poor prognosis in gastric cancer. PLoS One, 8, e81799 (2013).

\section{Figures}


A.

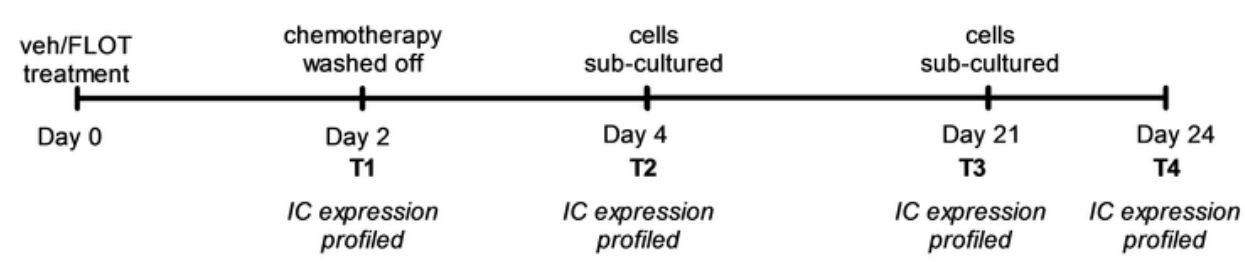

B.

IC ligands

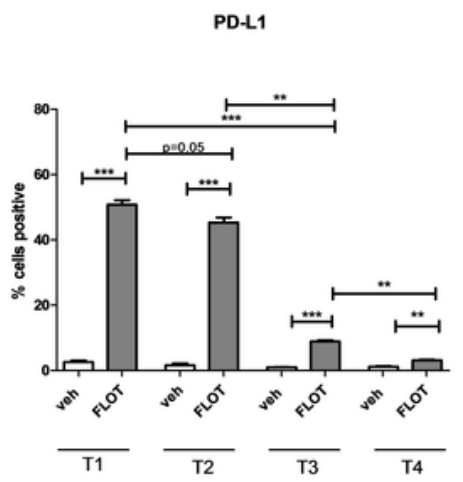

c.

PD-1

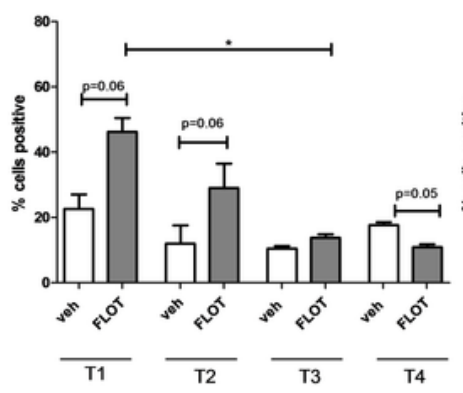

LAG-3

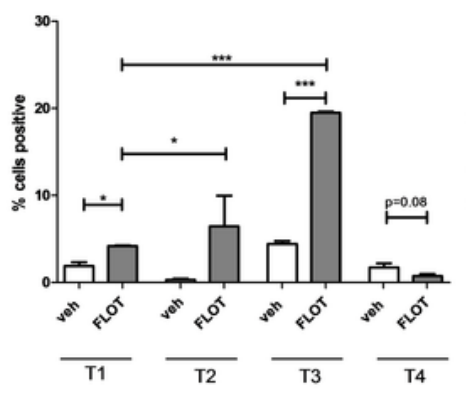

PD-L2

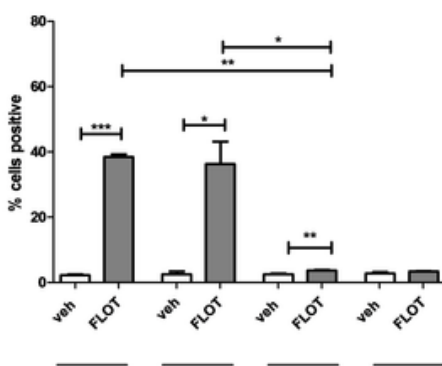

IC receptors
CD160

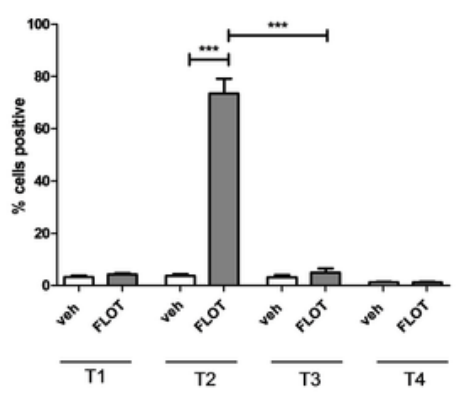

TIM-3
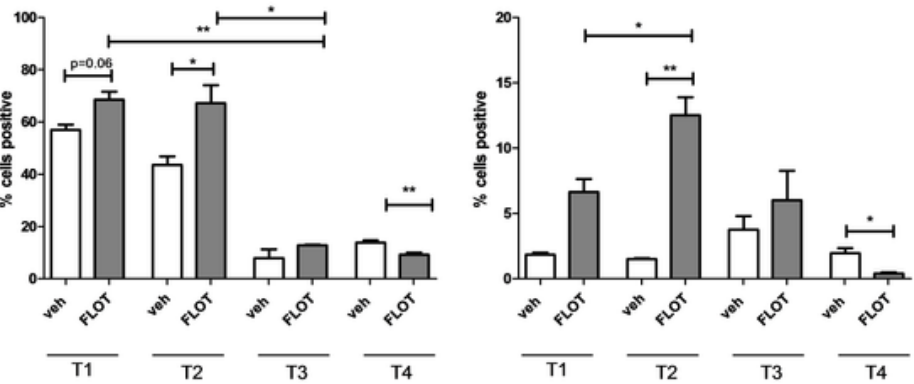

A2aR

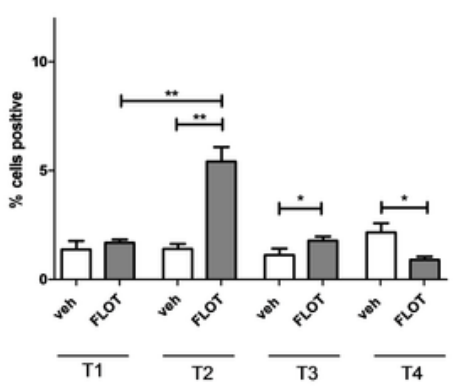

\section{Figure 1}

FLOT upregulates ICs on the surface of OAC cells in vitro, an effect which is maintained 3 weeks posttreatment. OE33 cells were treated with vehicle control (veh) or FLOT for 48h (T1), washed twice and allowed to grow for an additional 48h (T2) after which the cells were sub-cultured in new flasks and left to recover for 3 weeks (T3). Following complete recovery, the cells were sub-cultured 1 in 2 and screened for IC expression 3 days later (T4). IC ligand and receptor expression was profiled on the surface of OE33 
cells longitudinally by flow cytometry at $\mathrm{T} 1, \mathrm{~T} 2, \mathrm{~T} 3$ and $\mathrm{T} 4(\mathrm{n}=3)$. A) represents a schematic of experimental setup. B) IC ligands expression profiles for PD-L1, PD-L2 and CD160. C) IC receptor profiles for PD-1, TIGIT, TIM-3, LAG-3 and A2aR. Paired parametric t-test, ${ }^{*} \mathrm{p}<0.05,{ }^{* *} \mathrm{p}<0.01$ and ${ }^{* * *} \mathrm{p}<0.001$.

A.

PD-L1
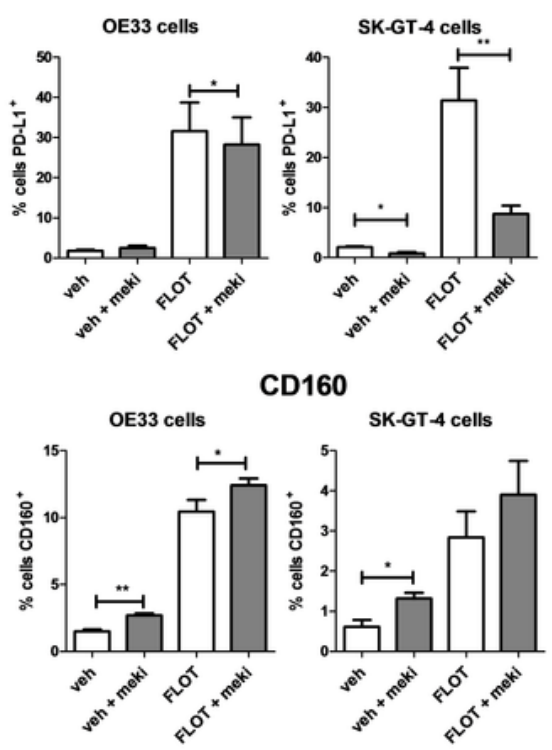

B.

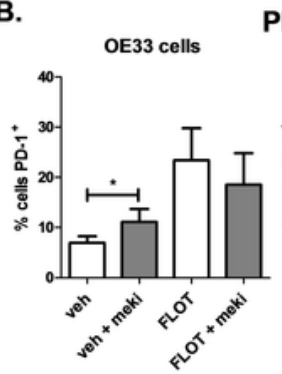

PD-1 SK-GT-4 cells

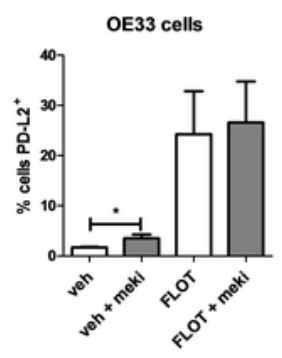

PD-L2
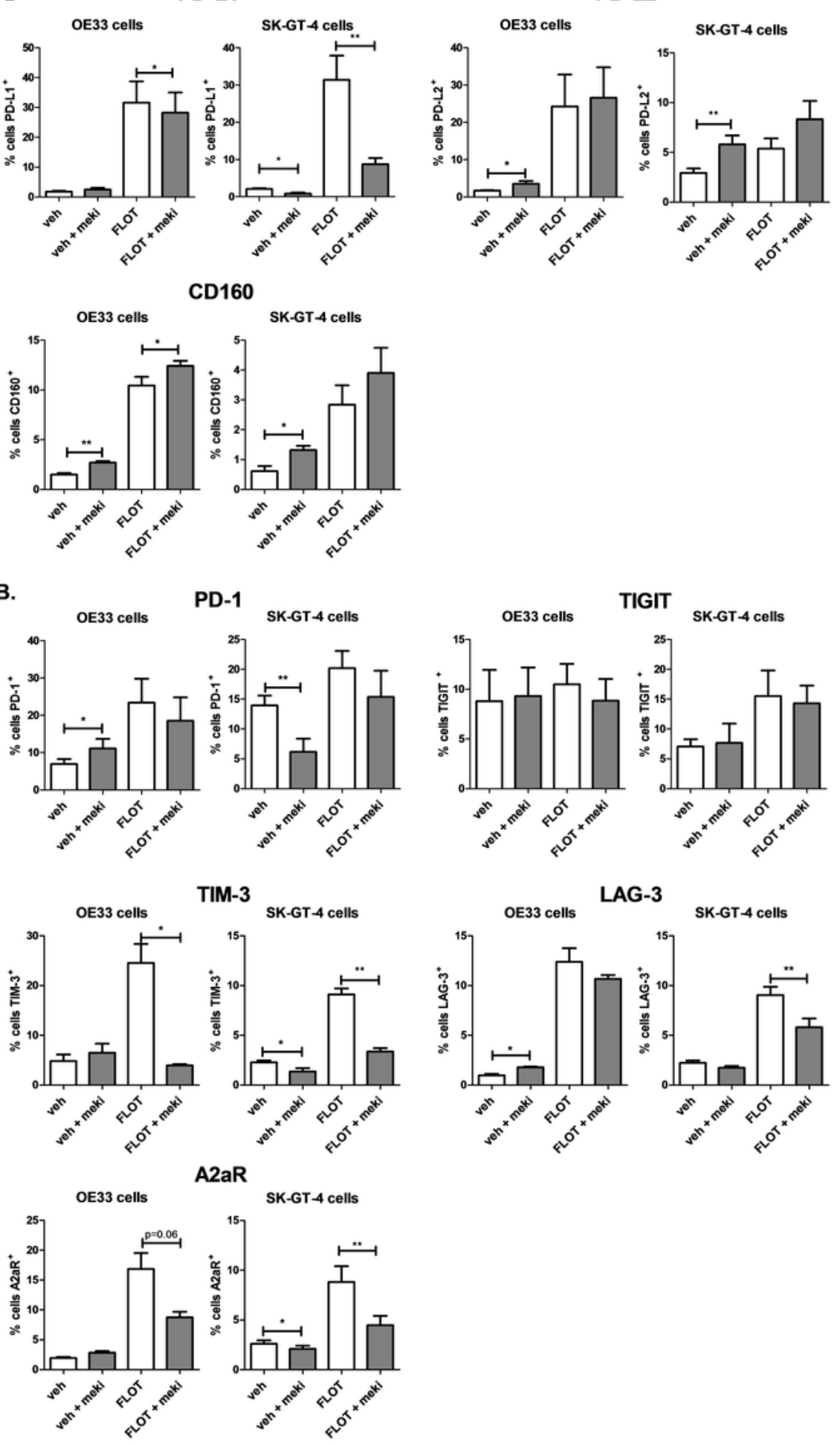

Figure 2

Inhibition of MEK signalling attenuates chemotherapy-induced upregulation of PD-L1, TIM-3, LAG-3 and A2aR on the surface of OAC cells in vitro. OE33 cells and SK-GT-4 cells were treated with and without 
FLOT chemotherapy regimen in the absence and presence of a MEK inhibitor (meki) for $48 \mathrm{~h}$ and the expression of IC ligands (PD-L1, PD-L2 and CD160) and IC receptors (PD-1, TIGIT, TIM-3, LAG-3 and A2aR) on the surface of OAC cells was determined by flow cytometry. ${ }^{*} p<0.05$ and ${ }^{* *} p<0.01$, experiments repeated $n=3$ times, paired parametric t-test.

A.

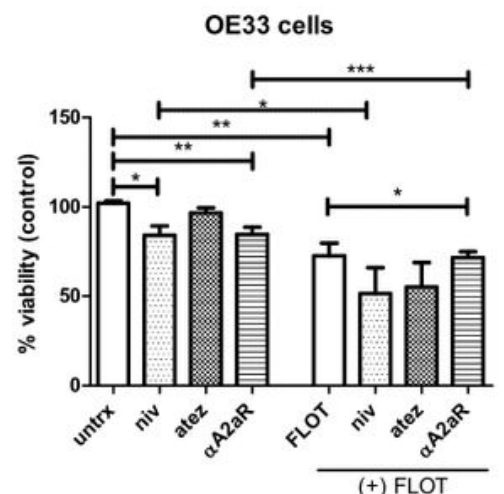

B.

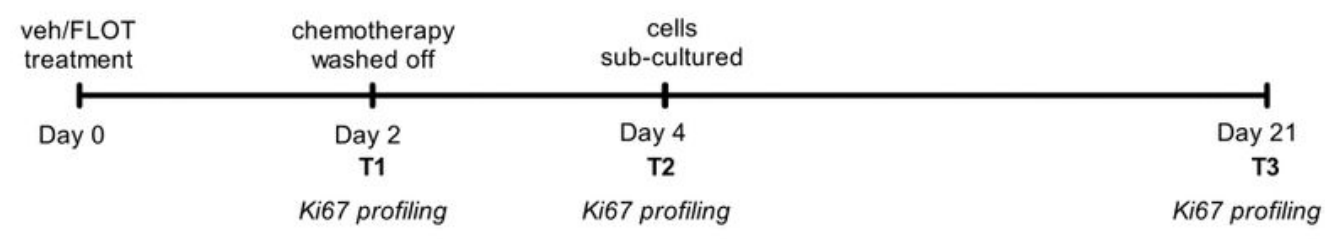

C.

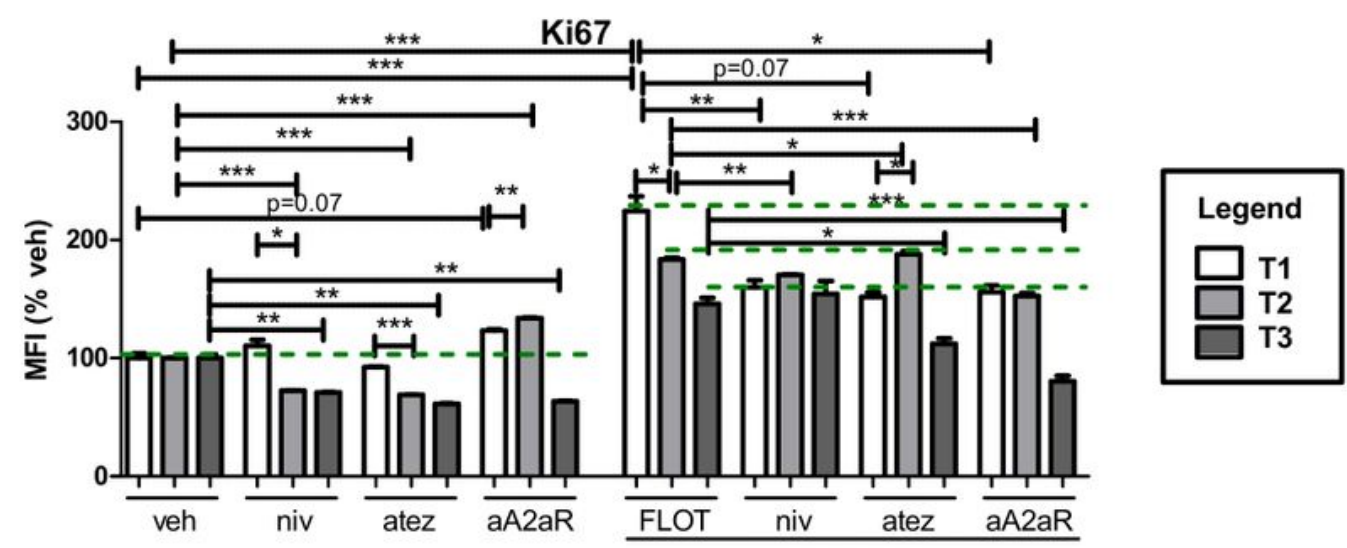

(+) FLOT

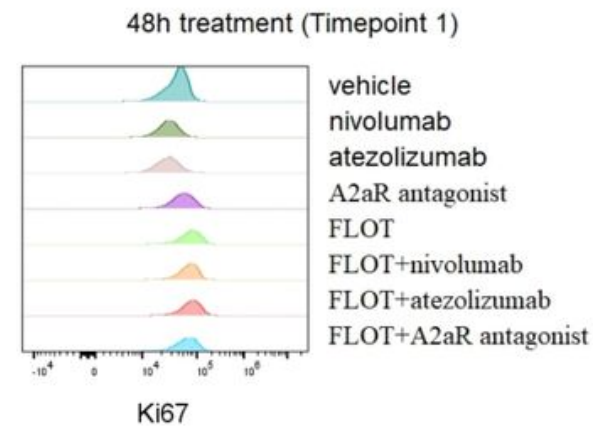

Figure 3

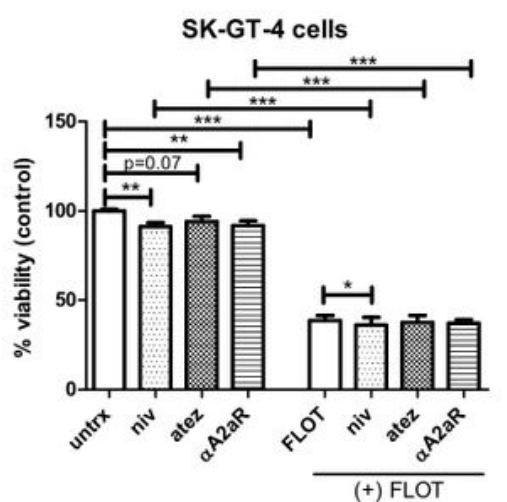

(+) FLOT 
Atezolizumab enhances the toxicity of FLOT chemotherapy regimen in OAC cells demonstrated by a significant decrease in viability and proliferation. OE33 cells and SK-GT-4 cells were treated with nivolumab $(10 \mu \mathrm{g} / \mathrm{ml})$, atezolizumab $(10 \mu \mathrm{g} / \mathrm{ml})$ or A2aR antagonist $(3 \mu \mathrm{M})$ in the absence or presence of FLOT regimen for $48 \mathrm{~h}$ and a CCK-8 assay (A). OE33 cells were treated with nivolumab $(10 \mu \mathrm{g} / \mathrm{ml})$, atezolizumab $(10 \mu \mathrm{g} / \mathrm{ml})$ or A2aR antagonist $(3 \mu \mathrm{M})$ in the absence or presence of FLOT regimen for $48 \mathrm{~h}$ (timepoint 1: T1), washed twice and allowed to grow for an additional 48h (T2) after which the cells were sub-cultured in new flasks and left to recover for 3 weeks (T3). Schematic representation of experimental setup depicted in (B). Ki67 mean fluorescence intensity (MFI) was assessed by intracellular flow cytometry in viable OE33 cells $(n=4)$. MFI expressed as a percentage of vehicle control \pm SEM.

Representative histograms displayed for each treatment at T1 and T3 showing effect of each treatment on Ki67 expression in OE33 cell line. Paired parametric t-test, ${ }^{*} p<0.05,{ }^{\star \star} p<0.01$ and ${ }^{* \star \star} p<0.001$. 


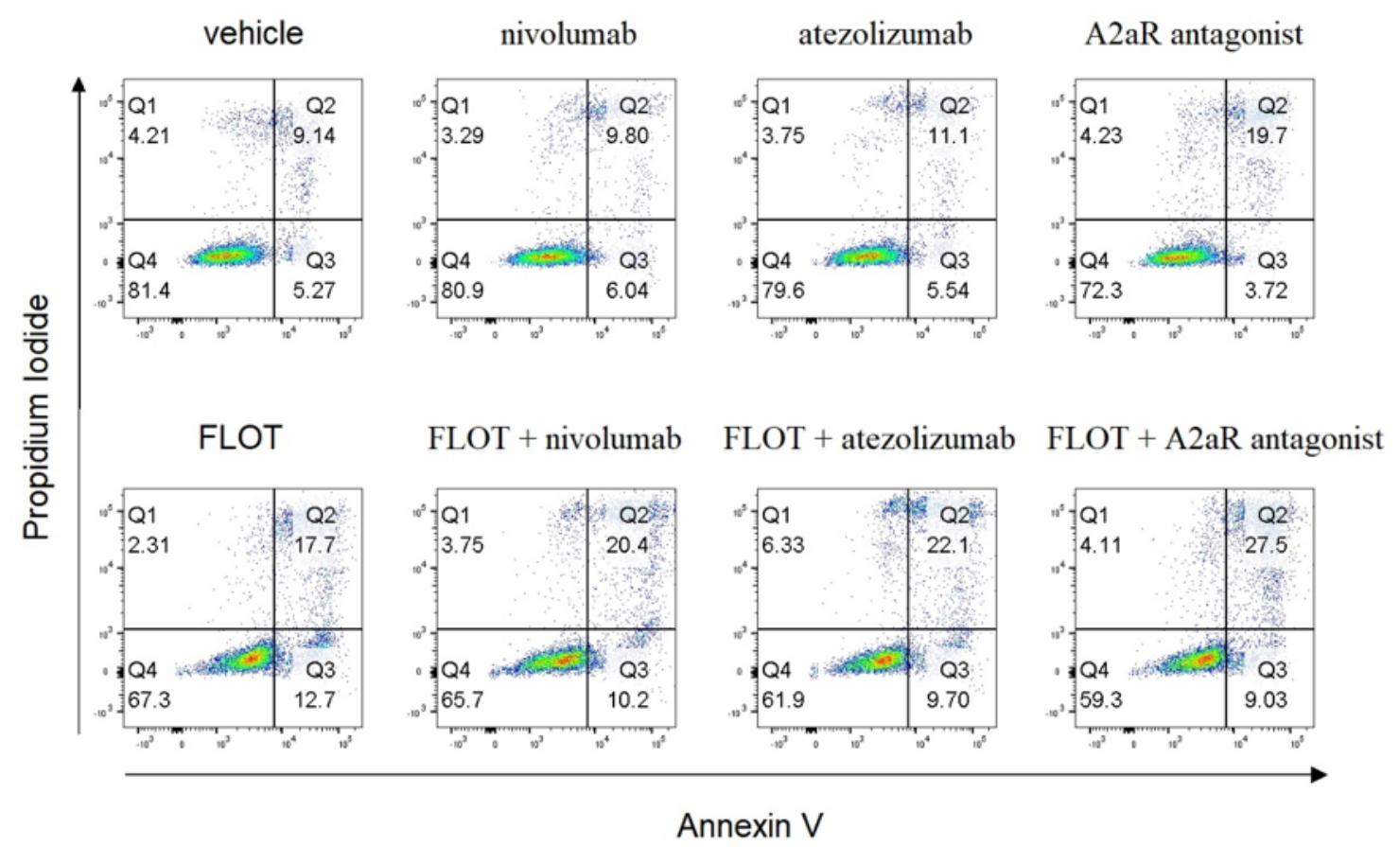

Necrotic cells

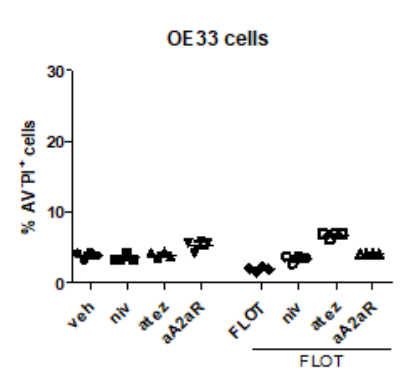

Viable cells

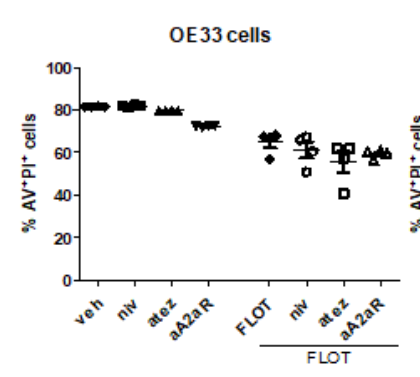

Late stage apoptotic cells
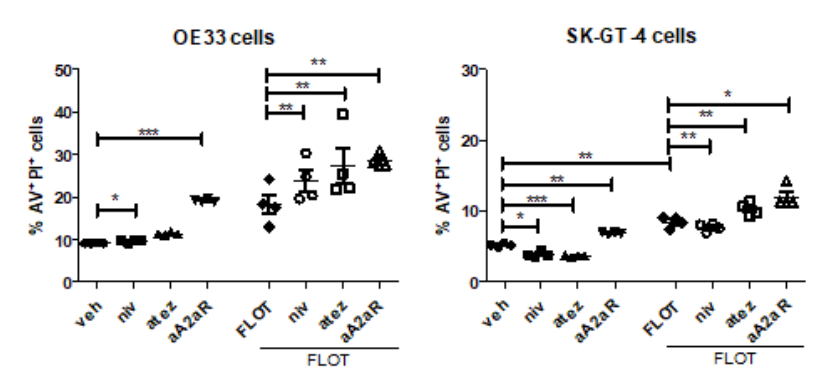

Early stage apoptotic cells
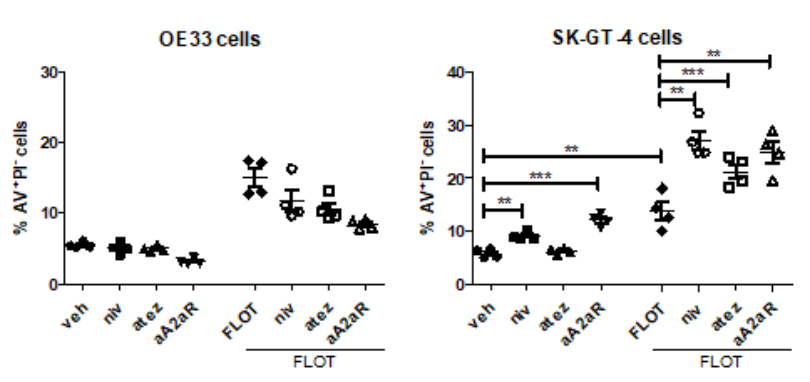

\section{Figure 4}

PD-1, PD-L1 and A2aR blockade enhances the toxicity of FLOT chemotherapy regimen demonstrated by a significant reduction in the percentage of viable cells and increase in late stage apoptotic cells. OE33 cells and SK-GT-4 cells were treated with nivolumab $(10 \mu \mathrm{g} / \mathrm{ml})$, atezolizumab $(10 \mu \mathrm{g} / \mathrm{ml})$ or A2aR antagonist $(3 \mu \mathrm{M})$ in the absence or presence of FLOT regimen for $48 \mathrm{~h}$. Viability was determined by flow cytometry using annexin $\mathrm{V}$ propidium iodide assay. Viable cells (AV-PI-), dead cells ( $\mathrm{PI}+$ ), apoptotic cells 
$(\mathrm{AV}+)$, early stage apoptotic cells ( $\mathrm{AV}+\mathrm{Pl}-$ ), late stage apoptotic cells ( $\mathrm{A} \mathrm{V}+\mathrm{PI}+)$ and necrotic cells ( $\mathrm{AV}-\mathrm{PI}+)$ were characterised. Representative dot plots shown for each treatment in OE33 cell line. Experiments repeated $n=4$ times. ${ }^{*} p<0.05,{ }^{* \star} p<0.01$ and ${ }^{* \star *} p<0.001$, paired parametric t-test.

A.
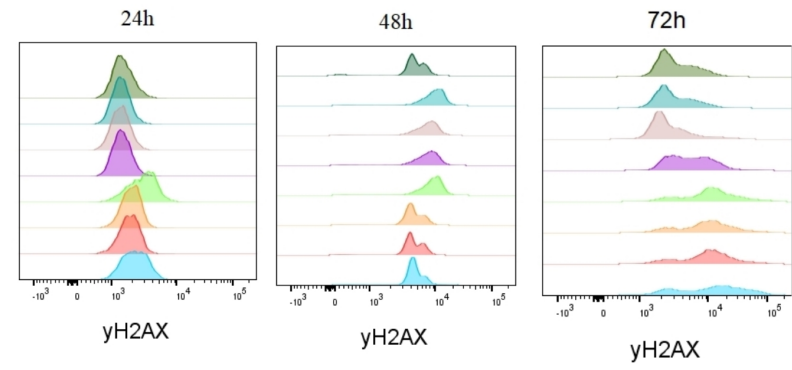

vehicle

nivolumab

atezolizumab

A2aR antagonist

FLOT + nivolumab

FLOT + atezolizumab

FLOT + A2aR antagonist
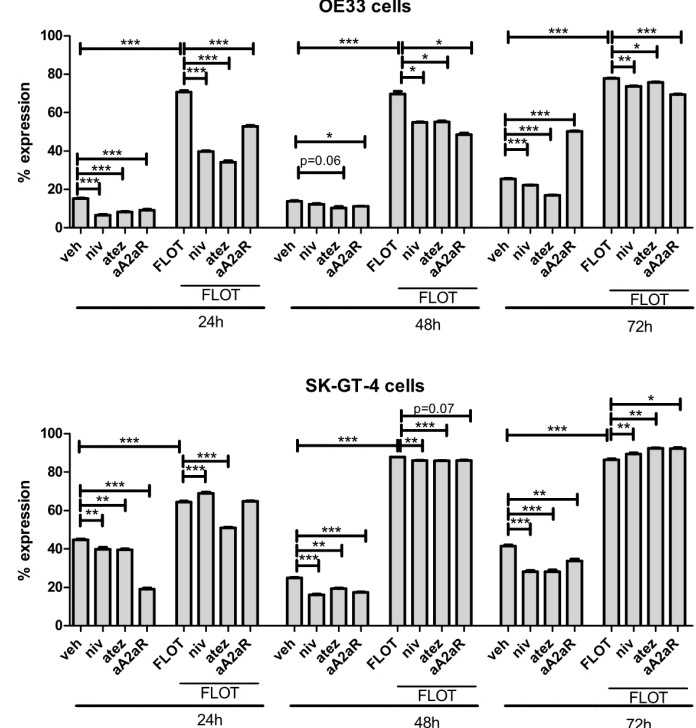

B.
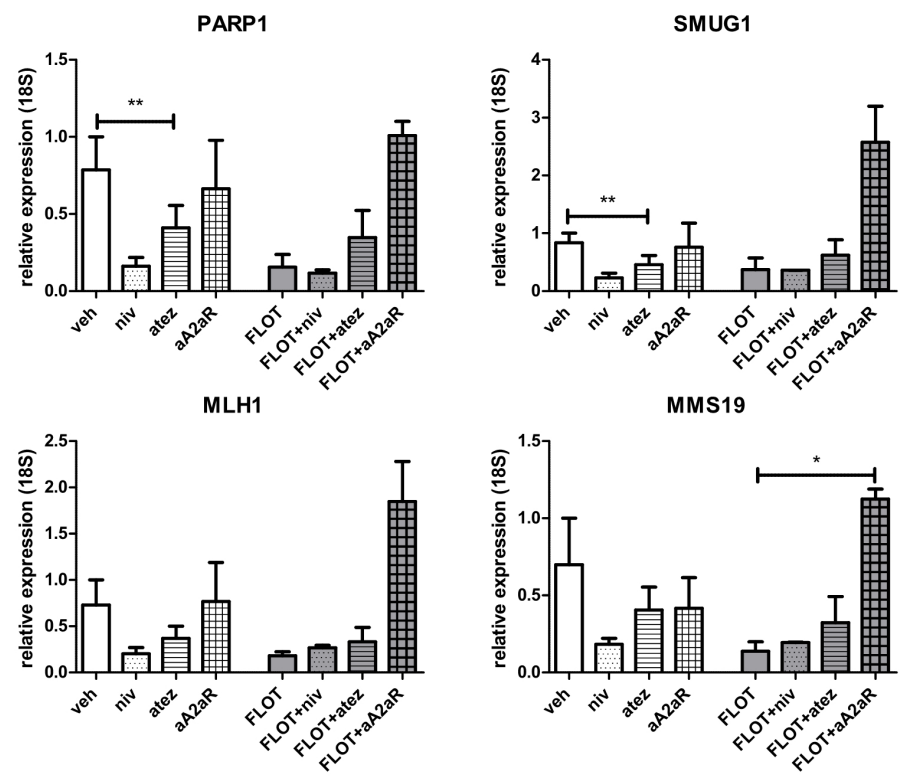

\section{Figure 5}

Single agent nivolumab and atezolizumab decreased the levels of $\mathrm{yH} 2 \mathrm{AX}$ and the levels of DNA repair genes in vitro. (A) OE33 cells and SK-GT-4 cells were treated with nivolumab $(10 \mu \mathrm{g} / \mathrm{ml})$, atezolizumab (10 
$\mu \mathrm{g} / \mathrm{ml})$ or A2aR antagonist $(3 \mu \mathrm{M})$ in the absence or presence of FLOT regimen for $24 \mathrm{~h}, 48 \mathrm{~h}$ and $72 \mathrm{~h}$. Expression of $\mathrm{\gamma H} 2 \mathrm{aX}$ was determined by intracellular flow cytometry. $\mathrm{\gamma H} 2 \mathrm{ax}$ expression is presented as mean fluorescence intensity (MFI). Representative histograms showing the levels of $\mathrm{YH} 2 \mathrm{AX}$ in $\mathrm{OE} 33$ cells for each treatment at $24 \mathrm{~h}, 48 \mathrm{~h}$ and $72 \mathrm{~h}$. (B) OE33 cells were treated with nivolumab $(10 \mu \mathrm{g} / \mathrm{ml})$, atezolizumab $(10 \mu \mathrm{g} / \mathrm{ml})$ or A2aR antagonist $(3 \mu \mathrm{M})$ in the absence or presence of FLOT regimen for $48 \mathrm{~h}$. mRNA expression levels of PARP1, SMUG1, MMS19 and MLH1 were determined by $\mathrm{qPCR}(\mathrm{n}=3$ in triplicate). Expression presented as relative quantity of $18 \mathrm{~S}$ housekeeping gene. ${ }^{*} p<0.05$ and ${ }^{* \star} p<0.01$, paired parametric t-test.
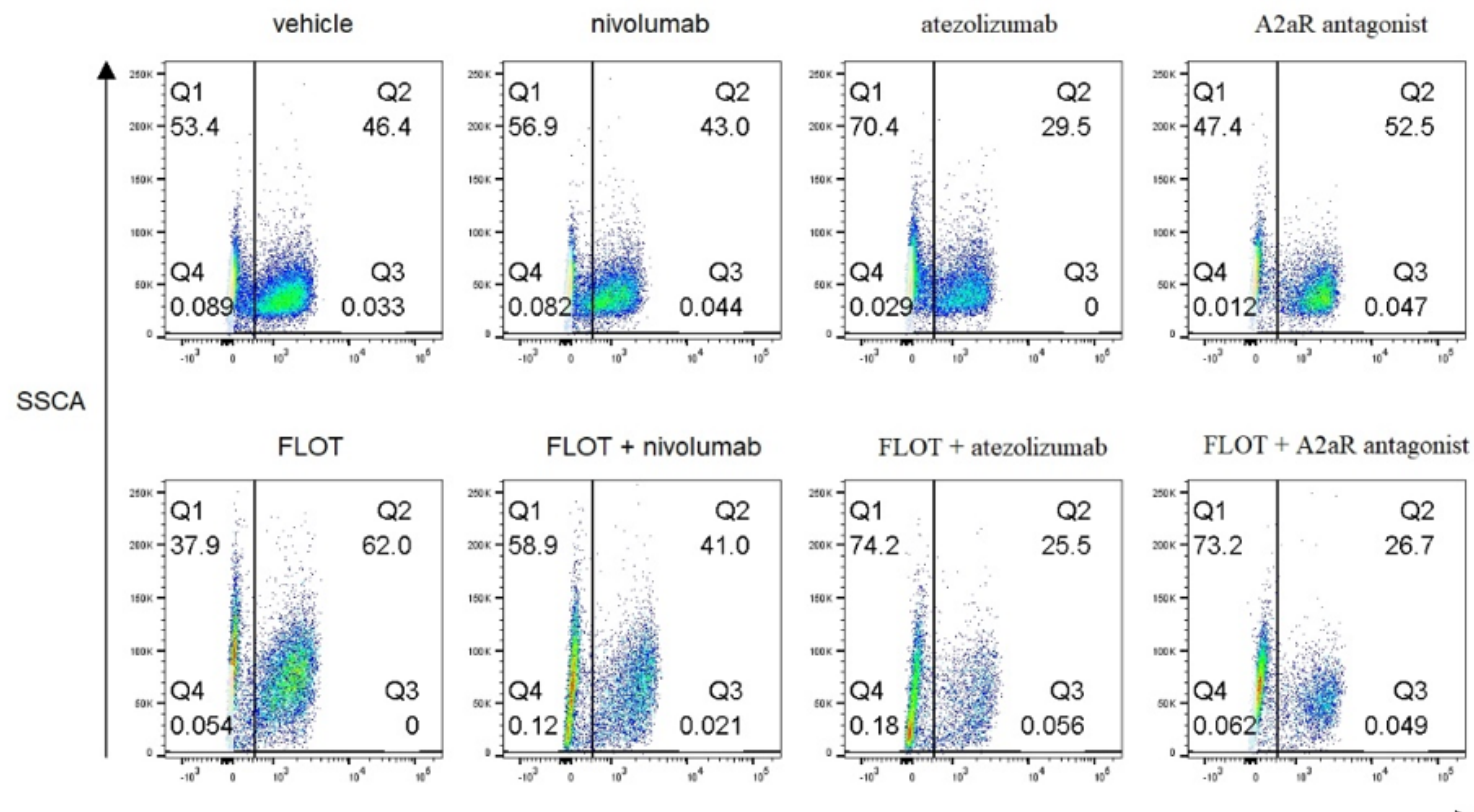

ALDH
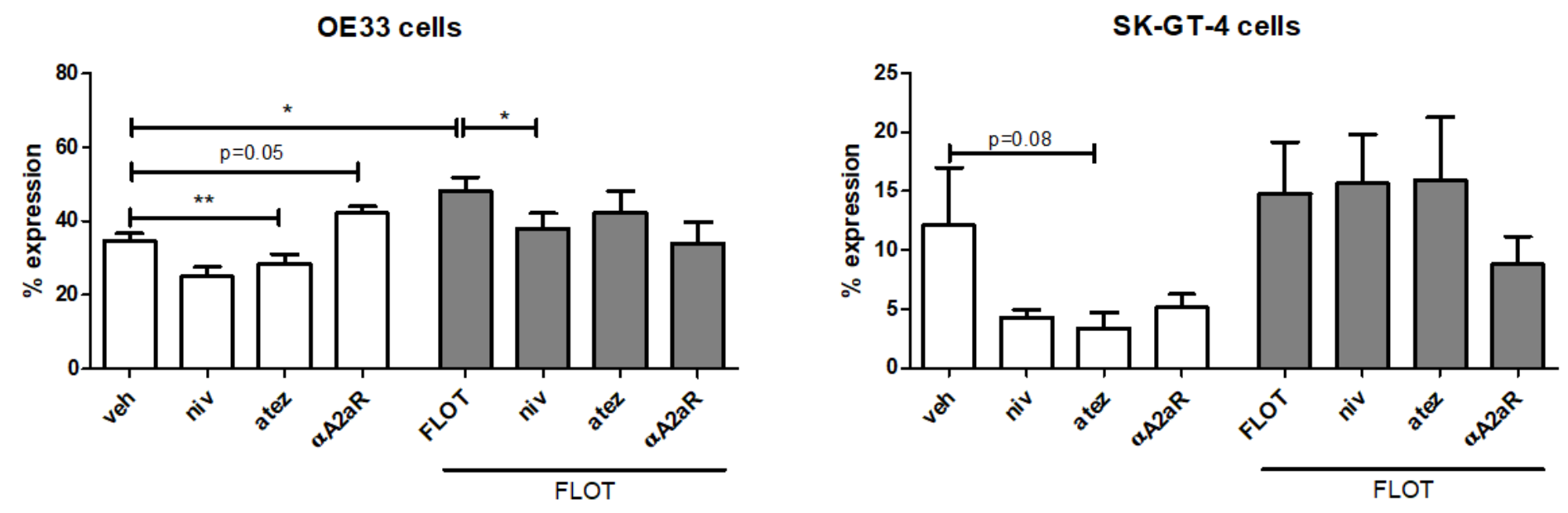

\section{Figure 6}

Nivolumab and atezolizumab treatment decrease the percentage of ALDH+ stem-like OAC cells in vitro. OE33 cells and SK-GT-4 cells were treated with nivolumab $(10 \mu \mathrm{g} / \mathrm{ml})$, atezolizumab $(10 \mu \mathrm{g} / \mathrm{ml})$ or A2aR antagonist $(3 \mu \mathrm{M})$ in the absence or presence of the FLOT regimen for $48 \mathrm{~h}$. ALDH activity was determined 
using an aldefluor assay by flow cytometry. Representative dot plots shown for each treatment. Experiments repeated $n=4$ times. ${ }^{*} p<0.05$ and ${ }^{* \star} p<0.01$, paired parametric t-test. 